\title{
A computational formulation for constrained solid and liquid membranes considering isogeometric finite elements
}

\author{
Roger A. Sauer 1 , Thang X. Duong, Callum J. Corbett \\ Aachen Institute for Advanced Study in Computational Engineering Science (AICES), RWTH Aachen \\ University, Templergaben 55, 52056 Aachen, Germany \\ Submitted on October $17^{\text {th }} 2012$ for publication in \\ Computer Methods in Applied Mechanics and Engineering
}

\begin{abstract}
A geometrically exact membrane formulation is presented that is based on curvilinear coordinates and isogeometric finite elements, and is suitable for both solid and liquid membranes. The curvilinear coordinate system is used to describe both the theory and the finite element equations of the membrane. In the latter case this avoids the use of local cartesian coordinates at the element level. Consequently, no transformation of derivatives is required. The formulation considers a split of the in-plane and out-of-plane membrane contributions, which allows the construction of a stable formulation for liquid membranes with constant surface tension. The proposed membrane formulation is general, and accounts for dead and live loading, as well as enclosed volume, area, and contact constraints. The new formulation is illustrated by several challenging examples, considering linear and quadratic Lagrange elements, as well as isogeometric elements based on quadratic NURBS and cubic T-splines. It is seen that the isogeometric elements are much more accurate than standard Lagrange elements. The gain is especially large for the liquid membrane formulation since it depends explicitly on the surface curvature.
\end{abstract}

Keywords: contact constraints, curvilinear coordinates, isogeometric analysis, nonlinear finite element methods, follower loads, volume constraints.

\section{Introduction}

Membranes are computationally challenging structures. Their geometry can be complex, they may undergo large deformations, large rotations and large strains - and thereby behave highly nonlinear - and they are characterized by several physical instabilities: They are unstable in compression, unstable for out-of-plane loading (in the case of zero in-plane tension), unstable for pressure loading (in the case of rubber membranes) and unstable w.r.t. in-plane loading (in the case of liquid membranes). The aim of this paper is to formulate a general, 3D, geometrically exact and fully nonlinear membrane model that accounts for pressure loading as well as volume, area, and contact constraints and is suitable for both solid and liquid membranes. Our focus is on pure membranes, i.e. curved, surface structures that do not support in-plane compression, out-of-plane bending, and shear ${ }^{2}$ Such a restricted focus is useful due to the large range of membrane applications: they appear as inflatable and pressurized structures, like balloons, tubes and airbags; as fabrics, tents, canopies, parachutes, foils and sails; as water-filled membrane

\footnotetext{
${ }^{1}$ corresponding author, email: sauer@aices.rwth-aachen.de

${ }^{2}$ We note that in the literature, the term membrane is often also used for the special case of $2 \mathrm{D}$ plane-stress structures.
} 
structures, like inflatable dams; as biological membranes, like blood vessels, cell, diaphragms, aneurysms and lung alveoli; as liquid droplets, menisci, bubbles, foams and sprays; as thin sheets and films - both liquid and solid - as atomistic membranes, like graphene sheets; as interacting membranes, e.g. adhering cells; and in the topic of form-finding and minimal surfaces.

Computational formulations for 3D, nonlinear membrane go back to the seminal work of Oden (Oden and Sato (1967), see also Oden (2006)). Since then, the field has been continuously advanced, among others by Fried (1982); Tang (1982); Roddeman et al. (1987); Contri and Schrefler (1988); Wriggers and Taylor (1990); Ibrahimbegovic and Gruttmann (1993); Haseganu and Steigmann (1994); Gosling and Lewis (1996); Muttin (1996); Wu et al. (1996); Bonet et al. (2000); Rumpel and Schweizerhof (2003); Stanuszek (2003); Weinberg and Neff (2008). Many of these works are concerned with the topic of wrinkling due to in-plane compression. More recently, computational formulations based on curvilinear coordinates have been considered rigorously, both for membranes (Ambroziak and Klosowski, 2006) and shells (Arciniega and Reddy, 2007). Another recent development are rotation-free shell formulations, as they have been considered by Flores and Estrada (2007); Linhard et al. (2007); Dung and Wells (2008) and recently Benson et al. (2011); Nguyen-Thanh et al. (2011) for isogeometric analysis. Isogeometric formulations allow the formulation of $C^{1}$-continuous surface formulations that are advantageous for flow simulations (Kiendl et al., 2010) and sliding contact (De Lorenzis et al., 2011; Temizer et al., 2012), see also Sauer $(2011,2012)$ for Hermite-based, $C^{1}$-continuous contact surfaces. Relevant to membranes is also the topic of live pressure loading (Bufler, 1984; Schweizerhof and Ramm, 1984). Membranes are also an interesting subject in shape optimization (Bletzinger et al., 2005; Manh et al., 2011).

The presented formulation contains several merits and novelties: It allows a split between inplane and out-of-plane contributions, which is used to construct a new formulation for liquid membranes. It admits arbitrary elastic material models for solid and liquid membranes. It is based purely on displacement-based finite elements and can be used with any kind of such elements. It includes, in particular, isogeometric NURBS elements to capture the deforming surface geometry to high-accuracy, even for comparably coarse discretizations. It is straight forward to implement in an existing FE framework. It avoids the need to transform derivatives between configurations and avoids the use of local cartesian coordinate systems. Shells models are often formulated using local cartesian coordinate systems, as this allows using classical constitutive relations formulated in this manner (Wriggers, 2008). To our mind, there is no need for such a detour: The balance laws, kinematics, constitutive relations as well as the FE weak forms and corresponding FE arrays can all be formulated efficiently in the curvilinear coordinate system. The capabilities of the presented formulation are demonstrated by several challenging computational examples, considering pressure loading, inflation and contact.

The following section presents the theory of nonlinear membranes in the framework of curvilinear coordinates, considering pressure loading, volume, area and contact constraints. Sec. 3 proposes a straight-forward finite element implementation of the theory, and Sec. 4 presents several examples of solid and liquid membranes to illustrate the capabilities of the present formulation.

\section{Nonlinear membranes}

In this section, we summarize the theory of nonlinear membranes in the framework of curvilinear coordinates. The membrane kinematics, constitution and balance laws in strong and weak form are discussed, and various kinds of constraints are considered. 


\subsection{Surface description in curvilinear coordinates}

The membrane surface, denoted $\mathcal{S}$, is fully characterized by the parametric description

$$
\boldsymbol{x}=\boldsymbol{x}\left(\xi^{1}, \xi^{2}\right) .
$$

This corresponds to a mapping of the point $\left(\xi^{1}, \xi^{2}\right)$ in the parameter domain $\mathcal{P}$ to the material point $\boldsymbol{x} \in \mathcal{S}$. In the following, Greek letters are used to denote the two indices 1 and 2. Summation is then implied on repeated indices. The tangent vectors to coordinate $\xi^{\alpha}$ at point $\boldsymbol{x} \in \mathcal{S}$ are given by

$$
\boldsymbol{a}_{\alpha}=\frac{\partial \boldsymbol{x}}{\partial \xi^{\alpha}}, \quad \alpha=1,2
$$

The two vectors form a basis for the tangent plane of $\mathcal{S}$. In general, they are not orthonormal. This apparent drawback of the description is actually an advantage when it comes to the kinematical description. This turns out to be very straightforward, e.g. see Eq. (16). The basis at $\boldsymbol{x}$ is characterized by the metric tensor, that has the co-variant components

$$
a_{\alpha \beta}:=\boldsymbol{a}_{\alpha} \cdot \boldsymbol{a}_{\beta}
$$

From the inversion

$$
\left[a^{\alpha \beta}\right]:=\left[a_{\alpha \beta}\right]^{-1}
$$

we obtain the contra-variant components of the metric tensor. Explicitly, we have $a^{11}=$ $a_{11} / \operatorname{det} a_{\alpha \beta}, a^{12}=-a_{12} / \operatorname{det} a_{\alpha \beta}$ and $a^{22}=a_{22} / \operatorname{det} a_{\alpha \beta}$. With these a dual basis can be constructed: From the co-variant base vectors $\boldsymbol{a}_{\alpha}$ the contra-variant counterparts

$$
\boldsymbol{a}^{\alpha}:=a^{\alpha \beta} \boldsymbol{a}_{\beta}
$$

can be determined. We note that summation is implied on repeated indices. Note that $\boldsymbol{a}^{\alpha} \cdot \boldsymbol{a}^{\beta}=$ $a^{\alpha \beta}$ and $\boldsymbol{a}_{\alpha} \cdot \boldsymbol{a}^{\beta}=\delta_{\alpha}^{\beta}$, where $\delta_{\alpha}^{\beta}$ is the Kronecker symbol. The unit normal of $\mathcal{S}$ at $\boldsymbol{x}$ is given by

$$
\boldsymbol{n}=\frac{\boldsymbol{a}_{1} \times \boldsymbol{a}_{2}}{\left\|\boldsymbol{a}_{1} \times \boldsymbol{a}_{2}\right\|} .
$$

It can be shown that

$$
\left\|\boldsymbol{a}_{1} \times \boldsymbol{a}_{2}\right\|=\sqrt{\operatorname{det} a_{\alpha \beta}} .
$$

The bases $\left\{\boldsymbol{a}_{1}, \boldsymbol{a}_{2}, \boldsymbol{n}\right\}$ and $\left\{\boldsymbol{a}^{1}, \boldsymbol{a}^{2}, \boldsymbol{n}\right\}$ can then be used to decompose any vector $\boldsymbol{v}$ on $\mathcal{S}$, i.e.

$$
\boldsymbol{v}=v^{\alpha} \boldsymbol{a}_{\alpha}+v_{\mathrm{n}} \boldsymbol{n}=v_{\alpha} \boldsymbol{a}^{\alpha}+v_{\mathrm{n}} \boldsymbol{n},
$$

where $v_{\alpha}$ denote the co-variant, and $v^{\alpha}$ the contra-variant components of $\boldsymbol{v}$. The derivative of the tangent vectors is given by

$$
\boldsymbol{a}_{\alpha, \beta}=\frac{\partial \boldsymbol{a}_{\alpha}}{\partial \xi^{\beta}} .
$$

Further, we require the so-called co-variant derivative of $\boldsymbol{a}_{\alpha}$, which is defined by

$$
\boldsymbol{a}_{\alpha ; \beta}:=\boldsymbol{a}_{\alpha, \beta}-\Gamma_{\alpha \beta}^{\gamma} \boldsymbol{a}_{\gamma}
$$

where $\Gamma_{\alpha \beta}^{\gamma}$ are the Christoffel symbols of the second kind given by $\Gamma_{\alpha \beta}^{\gamma}=\boldsymbol{a}_{\alpha, \beta} \cdot \boldsymbol{a}^{\gamma}$. Introducing the identity tensor on $\mathcal{S}$

$$
\mathbf{1}=\boldsymbol{a}_{\alpha} \otimes \boldsymbol{a}^{\alpha}=\boldsymbol{a}^{\alpha} \otimes \boldsymbol{a}_{\alpha}=\tilde{\mathbf{1}}-\boldsymbol{n} \otimes \boldsymbol{n},
$$


where $\tilde{\mathbf{1}}$ is the usual identity tensor in $\mathbb{R}^{3}, 3^{3}$ we can write

$$
\boldsymbol{a}_{\alpha ; \beta}=(\boldsymbol{n} \otimes \boldsymbol{n}) \boldsymbol{a}_{\alpha, \beta} .
$$

Contracting with $\boldsymbol{n}$ then yields

$$
\boldsymbol{n} \cdot \boldsymbol{a}_{\alpha ; \beta}=\boldsymbol{n} \cdot \boldsymbol{a}_{\alpha, \beta}=b_{\alpha \beta}
$$

which are the co-variant components of the curvature tensor $\boldsymbol{b}=b_{\alpha \beta} \boldsymbol{a}^{\alpha} \otimes \boldsymbol{a}^{\beta}$. The eigenvalues of this tensor are the principal curvatures of surface $\mathcal{S}$.

\section{$2.2 \quad$ Membrane kinematics}

Next, we consider the deformation of the membrane surface. We therefore distinguish between the deformed, current configuration $\mathcal{S}$ and the undeformed, initial configuration $\mathcal{S}_{0}$, see Fig. 1 . Both surfaces are described by the relations of Sec. 2.1. For surface $\mathcal{S}$ we use the lower case

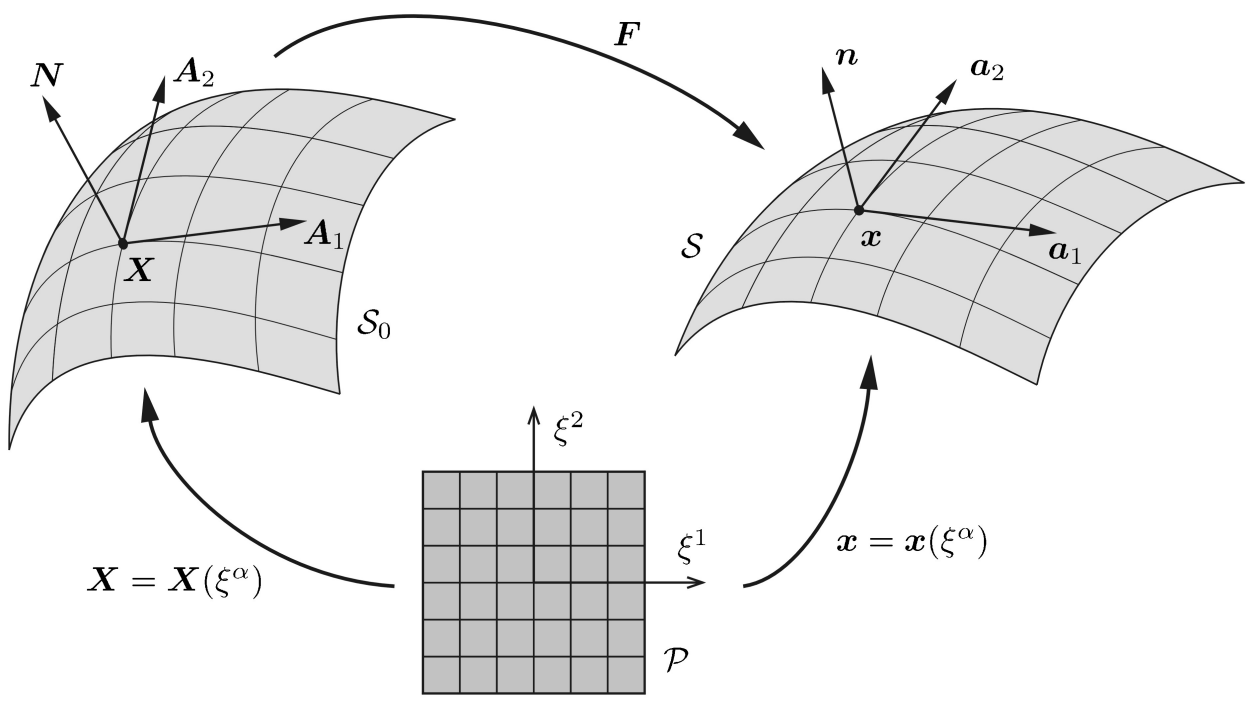

Figure 1: Mapping between parameter domain $\mathcal{P}$, reference surface $\mathcal{S}_{0}$ and current surface $\mathcal{S}$

symbols $\boldsymbol{x}, \boldsymbol{a}_{\alpha}, a_{\alpha \beta}, \boldsymbol{a}^{\alpha}, \boldsymbol{n}$ and $b_{\alpha \beta}$. For surface $\mathcal{S}_{0}$ we use the corresponding upper case symbols $\boldsymbol{X}, \boldsymbol{A}_{\alpha}, A_{\alpha \beta}, \boldsymbol{A}^{\alpha}$ and $\boldsymbol{N}$ In order to characterize the deformation between surfaces $\mathcal{S}_{0}$ and $\mathcal{S}$ consider the line element

$$
\mathrm{d} \boldsymbol{x}=\frac{\partial \boldsymbol{x}}{\partial \xi^{\alpha}} \mathrm{d} \xi^{\alpha}=\boldsymbol{a}_{\alpha} \mathrm{d} \xi^{\alpha}
$$

and likewise $\mathrm{d} \boldsymbol{X}=\boldsymbol{A}_{\alpha} \mathrm{d} \xi^{\alpha}$. Contracting with $\boldsymbol{A}^{\beta}$ yields d $\xi^{\alpha}=\boldsymbol{A}^{\alpha} \cdot \mathrm{d} \boldsymbol{X}$, so that

$$
\mathrm{d} \boldsymbol{x}=\left(\boldsymbol{a}_{\alpha} \otimes \boldsymbol{A}^{\alpha}\right) \mathrm{d} \boldsymbol{X} .
$$

Here the tensor

$$
\boldsymbol{F}=\boldsymbol{a}_{\alpha} \otimes \boldsymbol{A}^{\alpha}
$$

\footnotetext{
${ }^{3} \mathrm{~A}$ tilde is used here to indicate standard tensors in $\mathbb{R}^{3}$

${ }^{4}$ Here, the curvature tensor $\left[b_{\alpha \beta}\right]$ is only needed on $\mathcal{S}$.
} 
is the surface deformation gradient of the mapping $\boldsymbol{X} \rightarrow \boldsymbol{x}$. Likewise we find $\boldsymbol{F}^{-1}=\boldsymbol{A}_{\alpha} \otimes \boldsymbol{a}^{\alpha}$. Through $\boldsymbol{F}$ we thus have the following transformations

$$
\begin{array}{lll}
\boldsymbol{a}_{\alpha}=\boldsymbol{F} \boldsymbol{A}_{\alpha}, & \boldsymbol{A}_{\alpha}=\boldsymbol{F}^{-1} \boldsymbol{a}_{\alpha}, \\
\boldsymbol{a}^{\alpha}=\boldsymbol{F}^{-T} \boldsymbol{A}^{\alpha}, & \boldsymbol{A}^{\alpha}=\boldsymbol{F}^{T} \boldsymbol{a}^{\alpha} .
\end{array}
$$

Given $\boldsymbol{F}$, we can introduce the right and left Cauchy-Green surface tensors and their inverses, i.e.

$$
\begin{array}{lll}
\boldsymbol{C}=\boldsymbol{F}^{T} \boldsymbol{F}=a_{\alpha \beta} \boldsymbol{A}^{\alpha} \otimes \boldsymbol{A}^{\beta}, & \boldsymbol{C}^{-1}=a^{\alpha \beta} \boldsymbol{A}_{\alpha} \otimes \boldsymbol{A}_{\beta}, \\
\boldsymbol{B}=\boldsymbol{F} \boldsymbol{F}^{T}=A^{\alpha \beta} \boldsymbol{a}_{\alpha} \otimes \boldsymbol{a}_{\beta}, & \boldsymbol{B}^{-1}=A_{\alpha \beta} \boldsymbol{a}^{\alpha} \otimes \boldsymbol{a}^{\beta} .
\end{array}
$$

Next we discuss the surface stretch between surfaces $\mathcal{S}_{0}$ and $\mathcal{S}$. The area element $\mathrm{d} a \subset \mathcal{S}$ is defined by

$$
\mathrm{d} a:=\left\|\left(\boldsymbol{a}_{1} \mathrm{~d} \xi^{1}\right) \times\left(\boldsymbol{a}_{2} \mathrm{~d} \xi^{2}\right)\right\|=\left\|\boldsymbol{a}_{1} \times \boldsymbol{a}_{2}\right\| \mathrm{d} \square,
$$

where $\mathrm{d} \square:=\mathrm{d} \xi^{1} \mathrm{~d} \xi^{2}$. A corresponding statement follows for $\mathrm{d} A \subset \mathcal{S}_{0}$. In view of Eq. (7) we thus have the relations

$$
\begin{aligned}
\mathrm{d} A & =J_{A} \mathrm{~d} \square, & J_{A} & :=\sqrt{\operatorname{det} A_{\alpha \beta}}, \\
\mathrm{d} a & =J_{a} \mathrm{~d} \square, & J_{a} & :=\sqrt{\operatorname{det} a_{\alpha \beta}}, \\
\mathrm{d} a & =J \mathrm{~d} A, & J & :=J_{a} / J_{A} .
\end{aligned}
$$

\subsection{Momentum balance for membranes}

From the balance of linear momentum the strong form equilibrium equation

$$
\boldsymbol{t}_{; \alpha}^{\alpha}+\boldsymbol{f}=\mathbf{0},
$$

at $\boldsymbol{x} \in \mathcal{S}$ can be obtained (Steigmann, 1999). Here $\boldsymbol{f}$ is a distributed surface force, that can be decomposed as

$$
\boldsymbol{f}=f_{\alpha} \boldsymbol{a}^{\alpha}+p \boldsymbol{n}=f^{\alpha} \boldsymbol{a}_{\alpha}+p \boldsymbol{n}
$$

where $f_{\alpha}$ and $f^{\alpha}$ are the co-variant and contra-variant in-plane components of $\boldsymbol{f}$ and $p$ is the out-of-plane pressure acting on $\mathcal{S}$. Further, $\boldsymbol{t}^{\alpha}$ denotes the internal traction acting on the internal surface $\perp \boldsymbol{a}^{\alpha}$. According to Cauchy's formula

$$
\boldsymbol{t}^{\alpha}=\boldsymbol{\sigma} \boldsymbol{a}^{\alpha}
$$

where $\boldsymbol{\sigma}$ denotes the Cauchy stress tensor at $\boldsymbol{x} \in \mathcal{S}$, which we consider to be symmetric. We emphasize that $\boldsymbol{t}^{\alpha}$ is not a physical traction since $\boldsymbol{a}^{\alpha}$ is usually not normalized. In general, the stress tensor takes the form

$$
\boldsymbol{\sigma}=\sigma^{\alpha \beta} \boldsymbol{a}_{\alpha} \otimes \boldsymbol{a}_{\beta}+\sigma^{3 \alpha}\left(\boldsymbol{n} \otimes \boldsymbol{a}_{\alpha}+\boldsymbol{a}_{\alpha} \otimes \boldsymbol{n}\right)+\sigma^{33} \boldsymbol{n} \otimes \boldsymbol{n} .
$$

For membranes it is typically assumed that $\sigma^{3 \alpha}=\sigma^{33}=0$, so that

$$
\boldsymbol{\sigma}=\sigma^{\alpha \beta} \boldsymbol{a}_{\alpha} \otimes \boldsymbol{a}_{\beta} .
$$

In this case we find that $\boldsymbol{t}^{\alpha}=\sigma^{\beta \alpha} \boldsymbol{a}_{\beta}$ such that the co-variant derivative of $\boldsymbol{t}^{\alpha}$ becomes

$$
\boldsymbol{t}_{; \alpha}^{\alpha}=\sigma_{; \alpha}^{\alpha \beta} \boldsymbol{a}_{\beta}+\sigma^{\beta \alpha} b_{\alpha \beta} \boldsymbol{n}
$$

according to Eq. (13). Equilibrium equation (21) thus decomposes into

$$
\begin{array}{ll}
\sigma_{; \alpha}^{\alpha \beta}+f^{\beta}=0, & \text { (in-plane equilibrium), } \\
\sigma^{\alpha \beta} b_{\alpha \beta}+p=0, & \text { (out-of-plane equilibrium). }
\end{array}
$$


To close the problem, the usual Dirichlet and Neumann boundary conditions

$$
\begin{aligned}
& \boldsymbol{u}=\overline{\boldsymbol{u}} \text { on } \partial_{u} \mathcal{S} \\
& \boldsymbol{t}=\overline{\boldsymbol{t}} \quad \text { on } \partial_{t} \mathcal{S}
\end{aligned}
$$

are considered on the membrane boundary $\partial \mathcal{S}=\partial_{u} \mathcal{S} \cup \partial_{t} \mathcal{S}$. Here, we suppose that the prescribed traction $\overline{\boldsymbol{t}}=\bar{t}^{\alpha} \boldsymbol{a}_{\alpha}$ is tangent to $\mathcal{S}$, since out-of-plane boundary forces, as well as out-of-plane line and point loads within the surface, lead to singularities in the membrane deformation and are therefore not considered in the present formulation. The traction on boundary $\partial_{t} \mathcal{S}$, according to Cauchy's formula, is given by

$$
t=\sigma m
$$

where $\boldsymbol{m}=m_{\alpha} \boldsymbol{a}^{\alpha}$ is the outward unit normal of $\partial_{t} \mathcal{S}$. It follows that $\boldsymbol{t}=m_{\alpha} \boldsymbol{t}^{\alpha}$.

\subsection{Membrane constitution}

The known 3D constitutive models can be adapted to the membrane. We therefore suppose a general elastic material relation of the form $\tilde{\boldsymbol{\sigma}}=\tilde{\boldsymbol{\sigma}}(\tilde{\boldsymbol{B}})$. For membranes it is useful to consider the decomposition $\tilde{\boldsymbol{B}}=\boldsymbol{B}+\lambda_{3}^{2}(\boldsymbol{n} \otimes \boldsymbol{n})$, where $\lambda_{3}$ is the out-of-plane stretch, and $\tilde{\boldsymbol{\sigma}}=\boldsymbol{\sigma} / t+\sigma_{33}(\boldsymbol{n} \otimes \boldsymbol{n})$, where $\boldsymbol{\sigma}$ is defined as the in-plane stress tensor (with units force per length) and $t=\lambda_{3} T$ denotes the current membrane thickness, for a given reference thickness $T$. Out of these considerations, a relation between the membrane quantities $\boldsymbol{B}$ and $\boldsymbol{\sigma}$ can be obtained. As an example we consider an incompressible Neo-Hooke material, given by

$$
\tilde{\boldsymbol{\sigma}}=\tilde{\mu} \tilde{\boldsymbol{B}}+q \tilde{\mathbf{1}},
$$

where $\tilde{\mu}$ is the shear modulus and $q$ denotes the Lagrange multiplier associated with the incompressibility constraint. For membranes, the model decomposes into

$$
\begin{aligned}
& \boldsymbol{\sigma}=(\tilde{\mu} \boldsymbol{B}+q \mathbf{1}) T / \lambda_{3}, \\
& \sigma_{33}=\tilde{\mu} \lambda_{3}^{2}+q .
\end{aligned}
$$

For incompressibility $\operatorname{det} \tilde{\boldsymbol{B}}=\left(J \lambda_{3}\right)^{2}=1$. Under the plane stress assumption $\sigma_{33}=0$, we then find $q=-\tilde{\mu} / J^{2}$ and consequently

$$
\boldsymbol{\sigma}=\mu / J\left(\boldsymbol{B}-\mathbf{1} / J^{2}\right),
$$

with $\mu:=\tilde{\mu} T$. Componentwise, in the $\boldsymbol{a}_{\alpha}$ basis, this becomes

$$
\sigma^{\alpha \beta}=\mu / J\left(A^{\alpha \beta}-a^{\alpha \beta} / J^{2}\right) .
$$

Contracting with $a_{\beta \gamma}$, the components $\sigma_{\beta}^{\alpha}$ and $\sigma_{\alpha \beta}$ can be obtained ${ }^{5}$

Another example are liquid, e.g. water, membranes governed by constant isotropic surface tension $\gamma$. In that case a constant stress tensor of the form

$$
\sigma_{\beta}^{\alpha}=\gamma \delta_{\beta}^{\alpha}
$$

is obtained. It can be seen that the in-plane equilibrium equation $(26,1)$ is only satisfied for $f^{\alpha}=0$. This implies that static water membranes cannot equilibrate in-plane loads, and are therefore unstable in-plane; a property that needs to be addressed in a computational formulation (see Sec. 3). The out-of-plane equation $(26,2)$ now yields

$$
2 H \gamma+p=0, \quad 2 H:=b_{\alpha}^{\alpha},
$$

which is the well known Young-Laplace equation. A prominent feature of liquid membranes is that they form distinct contact angles. This property is not addressed here.

\footnotetext{
${ }^{5}$ Due to the symmetry of $\sigma^{\alpha \beta}$ the ordering of indices does not matter in $\sigma_{\beta}^{\alpha}$, i.e. $\sigma_{\beta}^{\alpha}=\sigma_{\beta}^{\alpha}$.
} 


\subsection{Membrane weak form}

Next, we derive the weak form corresponding to equilibrium equation (21). Consider a kinematically admissible variation of $\mathcal{S}$, denoted $\boldsymbol{w} \in \mathcal{W}$, where $\mathcal{W}$ denotes a suitable space for $\boldsymbol{w}$. Contracting Eq. (21) with $\boldsymbol{w}$ and integrating over $\mathcal{S}$ yields

$$
\int_{\mathcal{S}} \boldsymbol{w} \cdot\left(\boldsymbol{t}_{; \alpha}^{\alpha}+\boldsymbol{f}\right) \mathrm{d} a=0 \quad \forall \boldsymbol{w} \in \mathcal{W}
$$

Considering $\boldsymbol{w}=w_{\alpha} \boldsymbol{a}^{\alpha}+w \boldsymbol{n}$, this expands into

$$
\int_{\mathcal{S}} w_{\alpha}\left(\sigma_{; \beta}^{\alpha \beta}+f^{\alpha}\right) \mathrm{d} a+\int_{\mathcal{S}} w\left(\sigma^{\alpha \beta} b_{\alpha \beta}+p\right) \mathrm{d} a=0 \quad \forall \boldsymbol{w} \in \mathcal{W}
$$

i.e. it splits into the in-plane and out-of-plane parts identified in Eq. 26). Such a split is useful if different approximation techniques are chosen for the in-plane and out-of-plane response. Using the divergence theorem for curved surfaces (Gurtin and Murdoch, 1975), the first in-plane term is rewritten into

$$
\begin{aligned}
\int_{\mathcal{S}} w_{\alpha} \sigma_{; \beta}^{\alpha \beta} \mathrm{d} a & =\int_{\mathcal{S}}\left(w_{\alpha} \sigma^{\alpha \beta}\right)_{; \beta} \mathrm{d} a-\int_{\mathcal{S}} w_{\alpha ; \beta} \sigma^{\alpha \beta} \mathrm{d} a \\
& =\int_{\partial \mathcal{S}} w_{\alpha} \sigma^{\alpha \beta} m_{\beta} \mathrm{d} s-\int_{\mathcal{S}} w_{\alpha ; \beta} \sigma^{\alpha \beta} \mathrm{d} a
\end{aligned}
$$

where $m_{\alpha}=\boldsymbol{m} \cdot \boldsymbol{a}_{\alpha}$ are the co-variant components of the unit normal $\boldsymbol{m}$ on the line $\partial \mathcal{S}$. Since $w^{\alpha}=0$ on $\partial_{u} \mathcal{S}$ and since $w_{\alpha} \sigma^{\alpha \beta} m_{\beta}=w_{\alpha} \bar{t}^{\alpha}$ on $\partial_{t} \mathcal{S}$ expression 36 thus becomes

$$
G_{\text {int }}-G_{\text {ext }}=0 \quad \forall \boldsymbol{w} \in \mathcal{W},
$$

where

$$
\begin{aligned}
G_{\text {int }} & :=\int_{\mathcal{S}} w_{\alpha ; \beta} \sigma^{\alpha \beta} \mathrm{d} a-\int_{\mathcal{S}} w \sigma^{\alpha \beta} b_{\alpha \beta} \mathrm{d} a, \\
G_{\text {ext }} & :=\int_{\mathcal{S}} w_{\alpha} f^{\alpha} \mathrm{d} a+\int_{\partial_{t} \mathcal{S}} w_{\alpha} \bar{t}^{\alpha} \mathrm{d} s+\int_{\mathcal{S}} w p \mathrm{~d} a,
\end{aligned}
$$

are the internal and external virtual work contribution due to variation $\boldsymbol{w}$. Considering $w_{\alpha}=0$ and $w=0$ subsequently, the weak form can be decomposed into the weak forms

$$
\begin{aligned}
\int_{\mathcal{S}} w_{\alpha ; \beta} \sigma^{\alpha \beta} \mathrm{d} a-\int_{\mathcal{S}} w_{\alpha} f^{\alpha} \mathrm{d} a-\int_{\partial_{t} \mathcal{S}} w_{\alpha} \bar{t}^{\alpha} \mathrm{d} s=0 & \forall w_{\alpha} \in \mathcal{W}_{\alpha} \quad \text { (in-plane), } \\
\int_{\mathcal{S}} w \sigma^{\alpha \beta} b_{\alpha \beta} \mathrm{d} a+\int_{\mathcal{S}} w p \mathrm{~d} a=0 & \forall w \in \mathcal{W}_{\mathrm{n}} \quad \text { (out-of plane). }
\end{aligned}
$$

Such a split is advantageous for the description of liquid membranes. Since liquid membranes are inherently unstable in-plane, they can be stabilized by providing additional stiffness via Eq. (40.1) without affecting the out-of-plane response. This is demonstrated in the examples of Sec. 4.4 and 4.5 .

Otherwise, considering

$$
w_{\alpha ; \beta}=\left(\boldsymbol{w} \cdot \boldsymbol{a}_{\alpha}\right)_{; \beta}=\boldsymbol{w}_{; \beta} \cdot \boldsymbol{a}_{\alpha}+w b_{\alpha \beta},
$$

the two terms of $G_{\text {int }}$ can be combined into

$$
G_{\text {int }}=\int_{\mathcal{S}} \boldsymbol{w}_{; \alpha} \cdot \sigma^{\alpha \beta} \boldsymbol{a}_{\beta} \mathrm{d} a
$$


It is noted that for this expression only single derivatives of variation $\boldsymbol{w}$ and configuration $\boldsymbol{x}$ are required, while in the decomposed formulation of Eq. (40) second derivatives of $\boldsymbol{x}$ appear. Introducing the surface Kirchhoff stress tensor $\boldsymbol{\tau}=J \boldsymbol{\sigma}$, which eliminates one $J$ from expression (32), the last equation can be rewritten into

$$
G_{\text {int }}=\int_{\mathcal{S}_{0}} \boldsymbol{w}_{; \alpha} \cdot \tau^{\alpha \beta} \boldsymbol{a}_{\beta} \mathrm{d} A .
$$

Within framework (38), both dead and live loading can be considered. This is discussed further in Sec. 3.2. Beforehand, we discuss several useful membrane constraints.

\subsection{Volume constraints}

The volume of the domain $\mathcal{D}$ enclosed by the membrane may be constrained. An example is a cell containing incompressible fluid. Formally, the volume constraint is written as

$$
g_{\mathrm{v}}:=V-V_{0}=0,
$$

where $V_{0}$ and $V$ denote the initial and current volumes enclosed by the initial and current membrane configurations. Since $\mathrm{d} v=\boldsymbol{x} \cdot \boldsymbol{n} \mathrm{d} a / 3$ and $\mathrm{d} V=\boldsymbol{X} \cdot \boldsymbol{N} \mathrm{d} A / 3$, these can be computed by the surface integration

$$
V=\frac{1}{3} \int_{\mathcal{S}} \boldsymbol{x} \cdot \boldsymbol{n} \mathrm{d} a, \quad V_{0}=\frac{1}{3} \int_{\mathcal{S}_{0}} \boldsymbol{X} \cdot \boldsymbol{N} \mathrm{d} A
$$

These expressions are valid for closed surfaces, and care has to be taken when modeling open membranes. In this case one must account for the volume contribution associated with the missing surface. Eq. (44) can be included in the formulation by the Lagrange multiplier method. The Lagrange multiplier associated with the volume constraint is the internal membrane pressure $p$.

Remark: We note that the governing equations (38) and (44) can be derived from a variational principle for conservative systems. This is the case for the constitutive models discussed in Sec. 2.4 and for pressure loading of closed membranes surfaces.

\subsection{Area constraints}

Another useful constraint is a constraint on the membrane surface area. For example, red blood cells are known to conserve the surface area during deformation (Kloeppel and Wall, 2011). Formally this is expressed as

$$
g_{\mathrm{a}}:=A-A_{0}=0,
$$

with

$$
A=\int_{\mathcal{S}} \mathrm{d} a, \quad A_{0}=\int_{\mathcal{S}_{0}} \mathrm{~d} A .
$$

The area constraint is not considered further in this paper. In principle, they can be treated in an equivalent manner to volume constraints.

\subsection{Contact constraints}

Contact is characterized by the impenetrability constraint

$$
g_{\mathrm{n}} \leq 0
$$


where

$$
g_{\mathrm{n}}:=\left(\boldsymbol{x}-\boldsymbol{x}_{\mathrm{p}}\right) \cdot \boldsymbol{n}_{\mathrm{p}}
$$

denotes the normal gap between the membrane point $\boldsymbol{x} \in \mathcal{S}$ and the surface $\Gamma$ of a neighboring obstacle. Here, the unit vector $\boldsymbol{n}_{\mathrm{p}}$ denotes the surface normal of $\Gamma$ at the point $\boldsymbol{x}_{\mathrm{p}}$, which is the solution of the minimum distance problem

$$
\boldsymbol{x}_{\mathrm{p}}(\boldsymbol{x})=\left\{\boldsymbol{y} \mid \min _{\forall \boldsymbol{y} \in \Gamma}(\boldsymbol{x}-\boldsymbol{y}) \text { for } \boldsymbol{x} \in \mathcal{S}\right\} .
$$

We note that this minimization can cause difficulties for complex surface geometries (Wriggers, 2006). Constraint (48) can be included in the membrane formulation by various methods. The simplest of these is the penalty formulation. In this case the contact traction $\boldsymbol{t}_{\mathrm{c}}$ acting at $\boldsymbol{x} \in \mathcal{S}$ is given by

$$
\boldsymbol{t}_{\mathrm{c}}= \begin{cases}-\epsilon_{\mathrm{n}} g_{\mathrm{n}} \boldsymbol{n}_{\mathrm{p}}, & g_{\mathrm{n}}<0, \\ \mathbf{0}, & g_{\mathrm{n}} \geq 0,\end{cases}
$$

where $\epsilon_{\mathrm{n}}$ is the penalty parameter. The contact forces contribute to virtual work balance (38). This can be expressed by including

$$
G_{\mathrm{c}}=-\int_{\mathcal{S}} \boldsymbol{w} \cdot \boldsymbol{t}_{\mathrm{c}} \mathrm{d} a
$$

on the right hand side of Eq. (38). For two deformable membranes in contact, weak form (38) must be satisfied for each membrane, and contribution $G_{\mathrm{c}}$ is added correspondingly to each weak form. To avoid a surface bias it is advantageous to treat both contact pairs equivalently as is done in the two-half-pass algorithm of Sauer and De Lorenzis $(2012)$. For some problems, like adhesion, the contact constraint is replaced by suitable constitutive contact laws of the form $\boldsymbol{t}_{\mathrm{c}}=\boldsymbol{t}_{\mathrm{c}}\left(g_{\mathrm{n}}\right)$, see Sauer and Li (2007).

\section{Finite element discretization}

The governing equations (38) and (44) are solved by the finite element (FE) method. The initial surface $\mathcal{S}_{0}$ is therefore discretized into a set of finite elements $\Omega_{0}^{e}$ that are defined by nodal points $\boldsymbol{X}_{I}$ or control points in the case of isogeometric FE. The deforming membrane is then described by the motion of the nodal points $\boldsymbol{X}_{I} \rightarrow \boldsymbol{x}_{I}$, which corresponds to a Lagrangian FE description. The deformed configuration of element $\Omega_{0}^{e}$ is denoted $\Omega^{e}$. Here we consider quadrilateral elements since these can be conveniently related to a master element in the parameter domain $\xi^{\alpha} \in[-1,1]$.

\subsection{Finite element interpolation}

Within elements $\Omega_{0}^{e}$ and $\Omega^{e}$, the geometry is approximated by the nodal interpolations

$$
\boldsymbol{X} \approx \boldsymbol{X}^{h}=\sum_{I} N_{I} \boldsymbol{X}_{I},
$$

and

$$
\boldsymbol{x} \approx \boldsymbol{x}^{h}=\sum_{I} N_{I} \boldsymbol{x}_{I},
$$

where $N_{I}=N_{I}\left(\xi^{1}, \xi^{2}\right)$ denotes the nodal shape function defined on the master element in parameter space. The summation is carried out over the $n_{\text {ne }}$ nodes of the element. Here, the 
following quadrilateral elements are considered: 4-noded linear Lagrange elements, 9-noded quadratic Lagrange elements, quadratic NURBS elements, and T-spline elements. In principle, any other element type can also be considered. For isogeometric elements the shape functions are constructed via the Bézier extraction operation (Borden et al., 2011; Scott et al., 2011). According to Eq. (2), the tangent vectors are thus approximated by

$$
\boldsymbol{a}_{\alpha} \approx \sum_{I} N_{I, \alpha} \boldsymbol{x}_{I}
$$

where $N_{I, \alpha}=\partial N_{I} / \partial \xi^{\alpha}$. Considering a Bubnov-Galerkin formulation the variation $\boldsymbol{w}$ is approximated in the same way as the deformation, i.e.

$$
\boldsymbol{w} \approx \sum_{I} N_{I} \boldsymbol{w}_{I}
$$

For shorthand notation, we rewrite Eqs. (53), (54) and (56)

$$
\boldsymbol{X} \approx \mathbf{N X}_{e}, \quad \boldsymbol{x} \approx \mathbf{N x}_{e}, \quad \boldsymbol{w} \approx \mathbf{N w}_{e},
$$

where $\mathbf{N}:=\left[N_{1} \tilde{\mathbf{1}}, \ldots, N_{I} \tilde{\mathbf{1}}, \ldots\right]$ is a $\left(3 \times 3 n_{\mathrm{ne}}\right)$ array with the usual identity tensor $\tilde{\mathbf{1}}$ and $\mathbf{X}_{e}, \mathbf{x}_{e}$ and $\mathbf{w}_{e}$ are vectors containing the stacked nodal values for the element ${ }^{6}$ In order to discretize the weak form we need to discretize $w_{\alpha}, w$ and $\boldsymbol{w}_{; \alpha}$. We find

$$
\begin{aligned}
& w_{\alpha} \approx \boldsymbol{w} \cdot \boldsymbol{a}_{\alpha}=\mathbf{w}_{e}^{T} \mathbf{N}^{T} \boldsymbol{a}_{\alpha}=\mathbf{w}_{e}^{T} \mathbf{N}^{T} \mathbf{N}_{, \alpha} \mathbf{x}_{e} \\
& w \approx \boldsymbol{w} \cdot \boldsymbol{n}=\mathbf{w}_{e}^{T} \mathbf{N}^{T} \boldsymbol{n} \\
& \boldsymbol{w}_{; \alpha} \approx \mathbf{N}_{, \alpha} \mathbf{w}_{e}
\end{aligned}
$$

where $\mathbf{N}_{, \alpha}:=\left[N_{1, \alpha} \tilde{\mathbf{1}}, \ldots N_{I, \alpha} \tilde{\mathbf{1}}, \ldots\right]$. Note that for a vector like $\boldsymbol{w}$, the co-variant derivative $\boldsymbol{w}_{; \alpha}$ coincides with the regular partial derivative $\boldsymbol{w}_{, \alpha}$. The surface normal $\boldsymbol{n}$ is given through definition (6) and approximation (55). According to (13), the components of the curvature tensor become

$$
b_{\alpha \beta} \approx \boldsymbol{n} \cdot \mathbf{N}_{, \alpha \beta} \mathbf{x}_{e} .
$$

With the above expressions, we further find

$$
w_{\alpha ; \beta} \approx \mathbf{w}_{e}^{T}\left(\mathbf{N}_{, \beta}^{T} \mathbf{N}_{, \alpha}+\mathbf{N}^{T}(\boldsymbol{n} \otimes \boldsymbol{n}) \mathbf{N}_{, \alpha \beta}\right) \mathbf{x}_{e}
$$

according to eq. 41].

\subsection{Discretized weak form}

The above expressions are now used to discretize the membrane weak form of Sec. 2.5. The surface integration is carried out over the element domains $\Omega^{e}$ and then summed over all $n_{\mathrm{el}}$ $\mathrm{FE}$ as

$$
G_{\mathrm{int}}=\sum_{e=1}^{n_{\mathrm{el}}} G_{\mathrm{int}}^{e}, \quad G_{\mathrm{ext}}=\sum_{e=1}^{n_{\mathrm{el}}} G_{\mathrm{ext}}^{e} .
$$

For the internal virtual work of eq. (43) we now have

$$
G_{\mathrm{int}}^{e}=\int_{\Omega^{e}} \boldsymbol{w}_{; \alpha} \cdot \tau^{\alpha \beta} \boldsymbol{a}_{\beta} \mathrm{d} A \approx \mathbf{w}_{e}^{T} \int_{\Omega^{e}} \mathbf{N}_{, \alpha}^{T} \tau^{\alpha \beta} \mathbf{N}_{, \beta} \mathrm{d} A \mathbf{x}_{e}
$$

\footnotetext{
${ }^{6}$ Non-italic discrete arrays $\mathbf{X}, \mathbf{x}, \mathbf{w}$ and $\mathbf{N}$ should not be confused with italic field variables $\boldsymbol{X}, \boldsymbol{x}, \boldsymbol{w}$ and $\boldsymbol{N}$.
} 
according to approximations $(55)$ and $(58)$. Writing $G_{\mathrm{int}}^{e}=\mathbf{w}_{e}^{T} \mathbf{f}_{\text {int }}^{e}$, we identify the internal FE force vector

$$
\mathbf{f}_{\text {int }}^{e}=\int_{\Omega^{e}} \mathbf{N}_{, \alpha}^{T} \tau^{\alpha \beta} \mathbf{N}_{, \beta} \mathrm{d} A \mathbf{x}_{e} .
$$

As noted in Eq. (39.1), the internal virtual work can be split into in-plane and out-of plane contributions. At the element level these are

$$
G_{\text {inti }}^{e}=\int_{\Omega_{0}^{e}} w_{\alpha ; \beta} \tau^{\alpha \beta} \mathrm{d} A, \quad G_{\text {into }}^{e}=-\int_{\Omega_{0}^{e}} w \tau^{\alpha \beta} b_{\alpha \beta} \mathrm{d} A,
$$

such that $G_{\text {int }}^{e}=G_{\text {inti }}^{e}+G_{\text {into }}^{e}$. In view of Eqs. (58), (59) and (60), the corresponding force vectors become

$$
\begin{aligned}
& \mathbf{f}_{\text {inti }}^{e}=\int_{\Omega_{0}^{e}} \tau^{\alpha \beta}\left(\mathbf{N}_{, \alpha}^{T} \mathbf{N}_{, \beta}+\mathbf{N}^{T}(\boldsymbol{n} \otimes \boldsymbol{n}) \mathbf{N}_{, \alpha \beta}\right) \mathrm{d} A \mathbf{x}_{e}, \\
& \mathbf{f}_{\text {into }}^{e}=-\int_{\Omega_{0}^{e}} \tau^{\alpha \beta} \mathbf{N}^{T}(\boldsymbol{n} \otimes \boldsymbol{n}) \mathbf{N}_{, \alpha \beta} \mathrm{d} A \mathbf{x}_{e} .
\end{aligned}
$$

For $G_{\text {ext }}$ we consider external loading of the form $\boldsymbol{f}=\boldsymbol{f}_{0} / J+p \boldsymbol{n}$, where $\boldsymbol{f}_{0}$ and $p$ are given loading parameters. The first corresponds to a dead force per reference area, the second to a live pressure. According to Eq. $(39,2)$ we then have

$$
G_{\mathrm{ext}}^{e}=\int_{\Omega_{0}^{e}} \boldsymbol{w} \cdot \boldsymbol{f}_{0} \mathrm{~d} A+\int_{\partial_{t} \Omega^{e}} \boldsymbol{w} \cdot \overline{\boldsymbol{t}} \mathrm{d} s+\int_{\Omega^{e}} w p \mathrm{~d} a
$$

which yields the external force vector

$$
\mathbf{f}_{\text {ext }}^{e}=\int_{\Omega_{0}^{e}} \mathbf{N}^{T} \boldsymbol{f}_{0} \mathrm{~d} A+\int_{\partial_{t} \Omega^{e}} \mathbf{N}^{T} \overline{\boldsymbol{t}} \mathrm{d} s+\int_{\Omega^{e}} \mathbf{N}^{T} p \boldsymbol{n} \mathrm{d} a .
$$

The original weak form (38) now yields the descretized version

$$
\mathbf{w}^{T}\left[\mathbf{f}_{\mathrm{int}}-\mathbf{f}_{\mathrm{ext}}\right]=\mathbf{0}
$$

where $\mathbf{f}_{\text {int }}$ and $\mathbf{f}_{\text {ext }}$ are obtained from the assembly of the corresponding elemental force vectors, and $\mathbf{w}$ is the kinematically admissible set of all nodal variations. These are zero for the nodes on the Dirichlet boundary $\partial_{u} \mathcal{S}$. For the remaining nodes, Eq. (68) implies

$$
\mathbf{f}:=\mathbf{f}_{\text {int }}-\mathbf{f}_{\mathrm{ext}}=\mathbf{0}
$$

which is the discretized equilibrium equation that needs to be solved for the unknown nodal positions $\mathbf{x}$; see Sec. 3.5 .

We note, that in this formulation no mapping of derivatives between master and current configuration is required. Also no introduction of local, cartesian bases are needed. The formulation thus is straight forward and efficient to implement.

\subsection{Contact contributions}

The proposed membrane model can be easily extended to include contact, provided a 3D contact algorithm is available. The contact contribution (52) simply yields the force vector

$$
\mathbf{f}_{\mathrm{c}}^{e}=-\int_{\Omega_{k}^{e}} \mathbf{N}^{T} \boldsymbol{t}_{\mathrm{c}} \mathrm{d} a,
$$

that needs to be included in Eq. (69). For details on the the FE implementation of Eq. 70 we refer to Sauer and De Lorenzis (2012). 


\subsection{Discretized volume constraint}

The volume, enclosed by the discretized membrane surface, is obtained as

$$
V=\frac{1}{3} \sum_{e=1}^{n_{\mathrm{el}}} \int_{\Omega^{e}} \boldsymbol{n}^{T} \mathbf{N} \mathrm{d} a \mathbf{x}_{e}
$$

according to Eq. (45). For the volume constraint, $g_{\mathrm{v}}=V-V_{0}=0, V_{0}$ can be considered as an externally prescribed volume, e.g. during inflation, or as the initial value of $V$.

\subsection{Solution method}

The volume constraint is included in the formulation by the Lagrange multiplier method. The Lagrange multiplier associated with the constraint is the pressure, $p$, acting on membrane. Combining (44) with (69) leads to the system

$$
\begin{aligned}
& \mathbf{f}(\mathbf{x}, p)=\mathbf{0}, \\
& g_{\mathrm{v}}(\mathbf{x})=0,
\end{aligned}
$$

that needs to be solved for the unknown nodal position $\mathbf{x}$ and pressure $p$. Due to the nonlinearities of the model, this is solved with Newton's method. Therefore, the linearization of $\mathbf{f}$ and $g_{\mathrm{v}}$ w.r.t. $\mathbf{x}$ and $p$ are needed. This is discussed in Appendix B.

\subsection{Hydrostatic pressure}

In some applications, the pressure $p$ may vary locally. In static examples this is typically due to gravity. An example is the hydrostatic pressure distribution in a water-filled membrane. In this case, we have

$$
p=p_{\mathrm{v}}+p_{\mathrm{h}},
$$

where $p_{\mathrm{h}}$ is the hydrostatic, height dependent, pressure and $p_{\mathrm{v}}$ is the pressure associated with the volume constraint. The former is simply written as

$$
p_{\mathrm{h}}=-\rho \boldsymbol{g} \cdot \boldsymbol{x}
$$

where $\rho$ is the density of the pressure causing medium, and $\boldsymbol{g}$ is the gravity vector 7 The value of $p_{\mathrm{v}}$ is then the (constant) datum pressure at the origin.

\subsection{Numerical quadrature}

In parameter space, each element is defined on the master domain $\xi^{\alpha} \in[-1,1], \alpha=1,2$. The integrals from above are mapped to the master domain using transformations 200 . Integration is then carried out with standard Gaussian quadrature on the master domain.

\footnotetext{
${ }^{7}$ typically $\boldsymbol{g}=-[0,0, g]^{T}$, where $g$ is the gravity constant
} 


\subsection{Monitoring compression}

Membranes do not support in-plane compression. The absence of physical bending stiffness leads to buckling of the structure, known as wrinkling in the case of membranes. To avoid membrane compression in our formulation during computations, we simply monitor the minimum principal stress

$$
\sigma_{\min }=\frac{I_{1}}{2}-\sqrt{\frac{I_{1}^{2}}{4}-I_{2}}
$$

where $I_{1}=\operatorname{tr} \boldsymbol{\sigma}=\sigma_{\alpha}^{\alpha}$ and $I_{2}=\operatorname{det} \boldsymbol{\sigma}=\operatorname{det} \sigma_{\beta}^{\alpha}$ are the two invariants of the surface stress tensor. We note that $\sigma_{\min }$ does not imply the automatic failure of the discretize membrane structure as some numerical bending stiffness may be present. More involved wrinkling criteria can be found in the literature, see Lu et al. (2001) and Youn and Lee (2006).

\section{Numerical examples}

The proposed membrane model is illustrated by several examples, considering both solid and liquid membranes under dead, pressure, and volume loading. Standard linear and quadratic Lagrange finite elements as well as quadratic NURBS and cubic T-spline finite elements, providing $C^{1}$ - and $C^{2}$-continuous surface descriptions, respectively, are used.

\subsection{Inflation of a spherical balloon}

We first consider the inflation of a spherical rubber balloon and use it for validation, since an analytical solution exists for this problem. The rubber behavior is described by the incompressible Neo-Hookean material model (29). The finite element model of the balloon, modeled as $1 / 8$ th of a sphere, is shown in Fig. 2 a. Appropriate boundary conditions are provided to main-
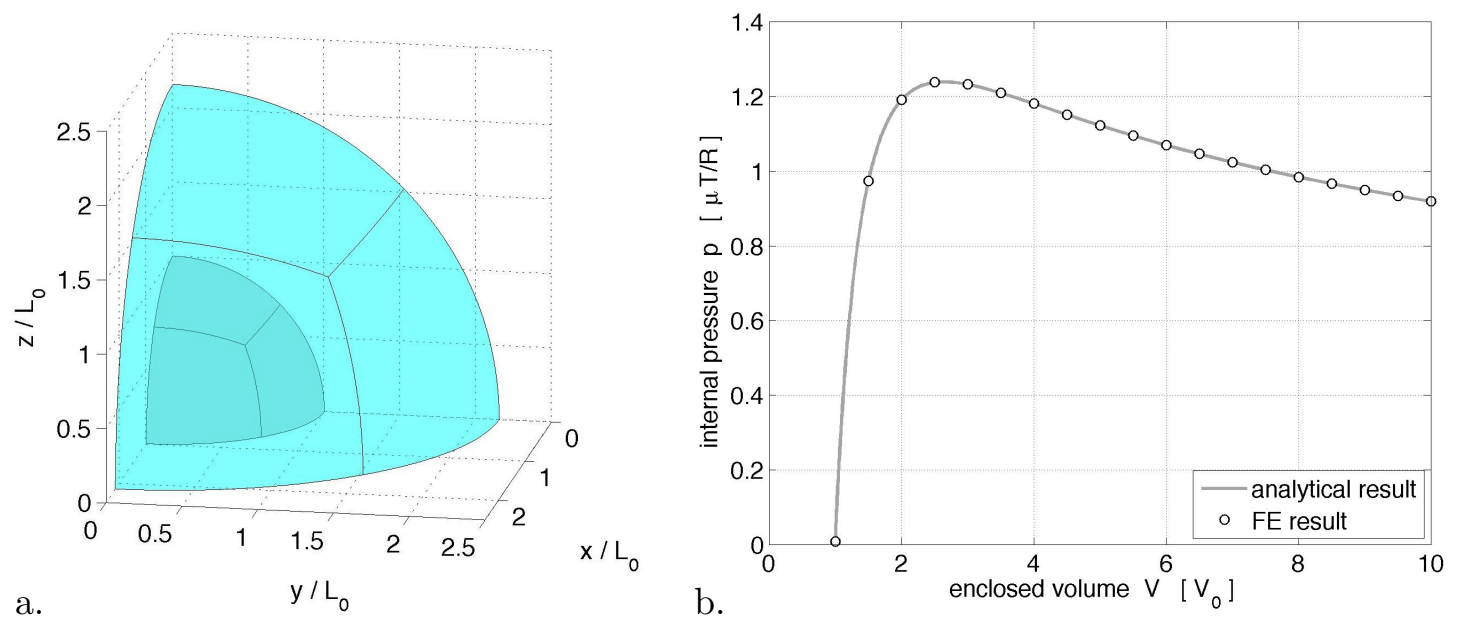

Figure 2: Inflated balloon: (a) initial and current configuration (for $V=10 V_{0}$ ); (b) pressurevolume relation for $V \in\left[\begin{array}{ll}1 & 10\end{array}\right] V_{0}$ (FE result for 3 quadratic FE).

tain the symmetry of the inflating structure. The relation between current and initial radius is denoted $r=\lambda R$. The circumference of the balloon, proportional to $r$, is thus stretched by $\lambda$ such that the surface deformation gradient is $\boldsymbol{F}=\lambda \mathbf{1}$ and the area change is given by $J=\lambda^{2}$. 
Due to incompressibility this results in the thickness change $t=T / J$. According to Eq. (31), the in-plane normal stress within the balloon thus is $\tilde{\sigma}=\sigma / t=\mu T\left(1-\lambda^{6}\right) / t$, which is equal to the well-known formula $\tilde{\sigma}=p r / 2 / t$. We thus obtain the pressure-stretch relation

$$
\frac{p R}{\mu T}=2\left(\frac{1}{\lambda}-\frac{1}{\lambda^{7}}\right)
$$

or, equivalently, the pressure-volume relation

$$
\frac{p R}{\mu T}=2\left(\left(\frac{V_{0}}{V}\right)^{\frac{1}{3}}-\left(\frac{V_{0}}{V}\right)^{\frac{7}{3}}\right)
$$

where $V_{0}=4 \pi / 3 R^{3}$ is the initial balloon volume. The $p-V$ relation is shown in Fig. $2 \mathrm{p}$. The pressure increases quickly, peaks and then decreases gradually. This behavior is typical for the inflation of rubber membranes. The FE computation of such problems should therefore be carried out by prescribed volume loading instead of prescribed pressure loading. The proposed FE formulation can capture the analytical behavior very nicely. This is shown by the convergence plot of Fig. 3a. Here, the number of Gaussian quadrature points per elements are

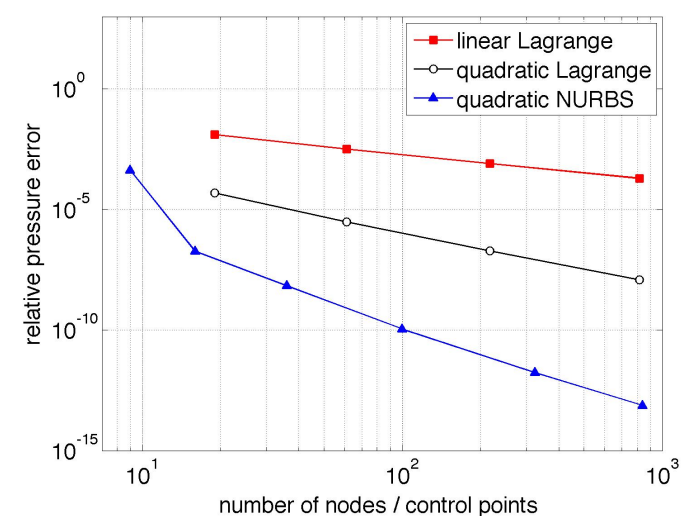

a.

Figure 3: Inflated balloon: convergence of the pressure at $V=10 V_{0}$ : (a) convergence with mesh size; (b) NURBS convergence with quadrature accuracy.

$2 \times 2$ for linear Lagrange and $3 \times 3$ for quadratic Lagrange and NURBS elements. Since NURBS elements describe the spherical geometry exactly, they can solve the problem exactly with only one element - provided sufficiently many quadrature points are used. This is shown in Fig. 3p. The results shown here validate the proposed membrane formulation.

Fig 4 shows the deformed FE meshes and the error in the membrane stress $\sigma$ for the three different element types considered here. As is seen, the error is smallest for NURBS FE.

\subsection{Inflation of a square sheet}

As a second example we consider a square membrane sheet with dimension $4 L_{0} \times 4 L_{0}$, apply an isotropic pre-stretch of $\lambda_{0}=1.05$ to provide initial out-of-plane stiffness, and then inflate the structure by a prescribed volume, as is shown in Fig. 5 .

Fig. 6a shows the pressure-volume relation for the three considered elements. The accuracy is highest for NURBS elements and lowest for linear elements. This is seen by the convergence behavior of the different element types, shown in Fig. 6b. 

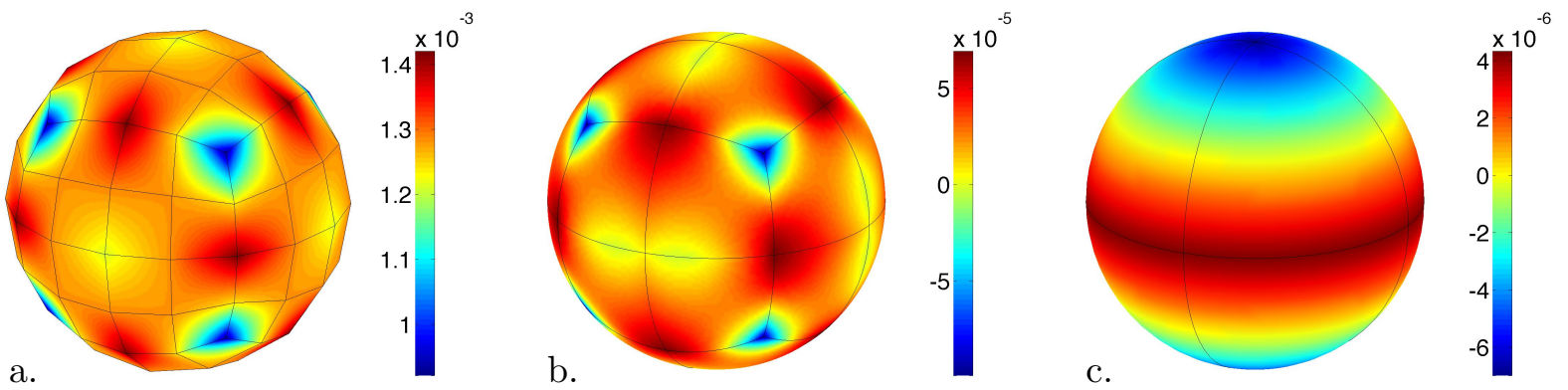

Figure 4: Inflated balloon: Error in the in-plane stress $\sigma=p r / 2$ for: (a) $12 \times 8$ linear FE, (b) $3 \times 8$ quadratic FE, (c) $1 \times 8$ NURBS FE.
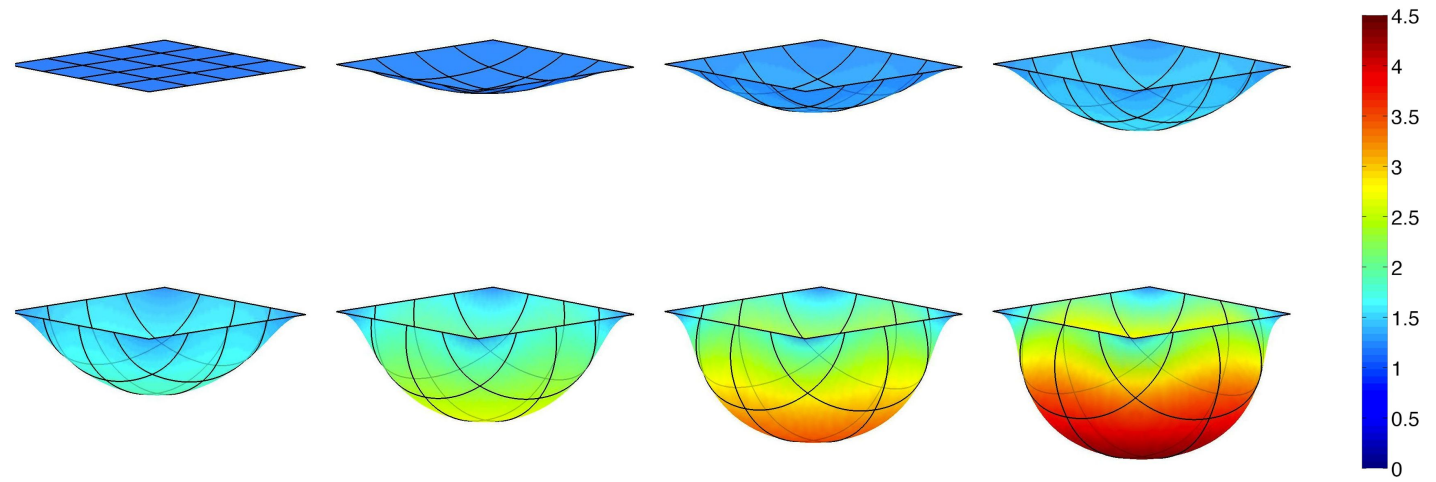

Figure 5: Inflated square sheet: configurations for $V=\{0,1,2,3,4,6,8,10\} V_{0}$, where $V_{0}=$ $4 L_{0}^{3}$. The coloring shows the area stretch $J$ (which is identical to the thickness decrease).

Fig. 7 shows the deformed sheet for a prescribed volume of $V=5000 V_{0}$ for the three element types. As seen, all element types can accommodate enormous deformations, even for relatively coarse meshes. The comparison with the fine NURBS mesh in Fig. 8a shows that there are still considerable inaccuracies present in all three formulations. The NURBS result is fully $C^{1}$-continuous. In the example, particularly large deformation occur at the bottom and in the corners of the sheet, as is seen in the close-up of Fig. $8 \mathrm{~b}$. The deformation in the corner shown a tendency towards wrinkling. We observed that a further mesh refinement led to non-convergent Newton behavior, indicating instabilities. A computational scheme for wrinkling is required to handle this case.

\subsection{Contact between balloon and cushion}

The third example considers a spherical, water-filled balloon in contact with a cushion. The balloon is loaded by hydrostatic pressure loading. The cushion is modeled by a square sheet that is fixed along the boundary and supported by internal pressure arising from constraining the volume beneath the sheet. The initial size of the sheet is $2 R \times 2 R$, where $R$ is the undeformed radius of the balloon. Both, balloon and sheet are modeled by material law 29 considering equal $\mu$. They are both pre-stretched isotropically by $\lambda_{0}=1.1$, i.e. the constrained balloon volume is $V_{0}=3 \pi(\lambda R)^{3} / 4$. The problem is computed by gradually increasing the gravity level, $g$, pulling on the water inside the balloon. Quadratic finite elements are used. Contact is modeled by the two-half-pass contact algorithm (Sauer and De Lorenzis, 2012) considering the 

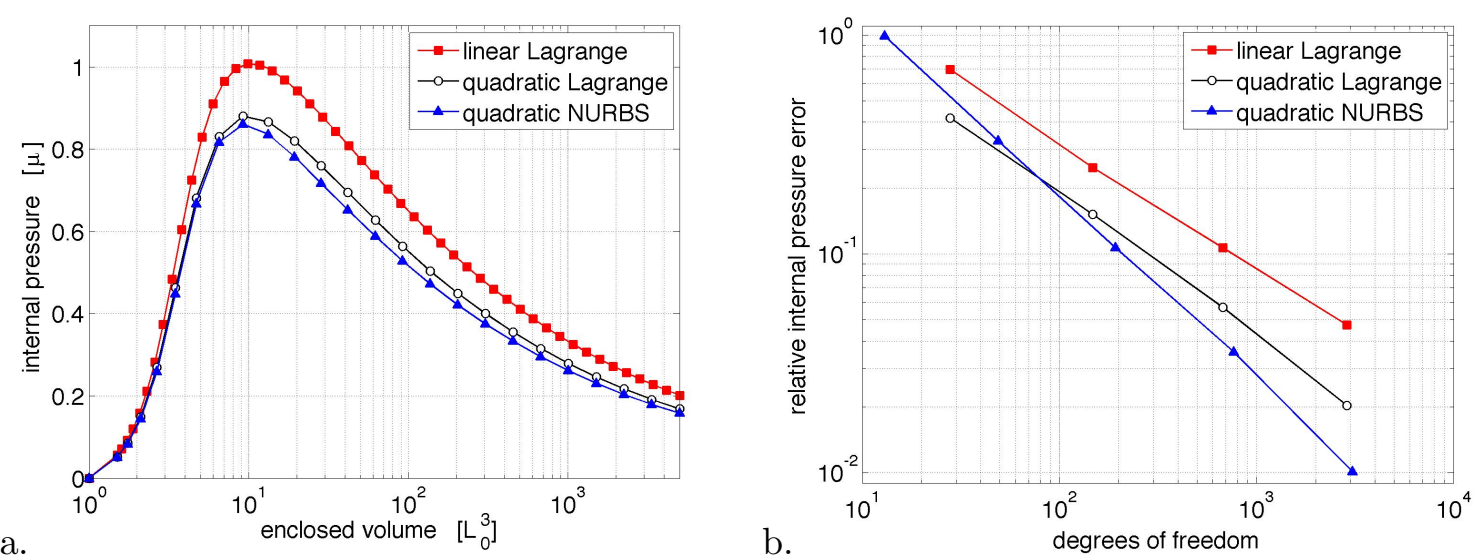

Figure 6: Inflated square sheet: (a) pressure-volume relation; (b) pressure convergence at $V=$ $5000 V_{0}$ (compared to a quadratic NURBS mesh with 23233 dofs).

a.

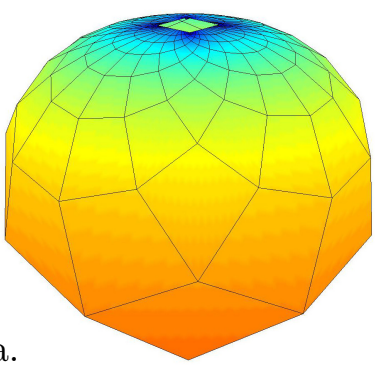

b.

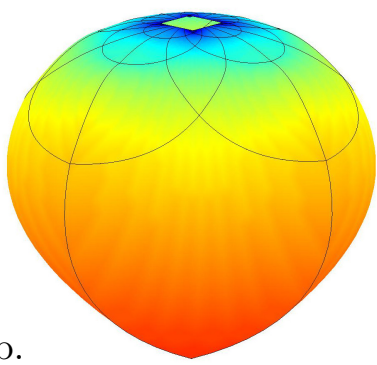

c.

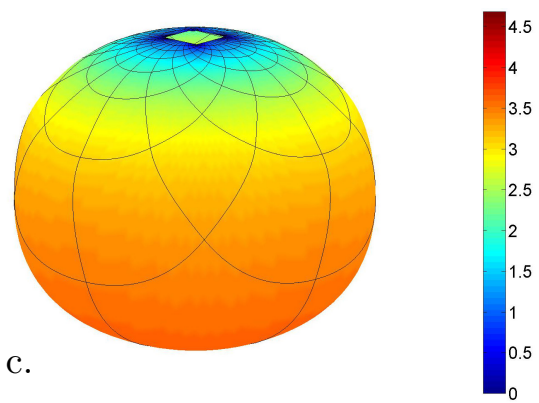

Figure 7: Inflated square sheet: deformation at $V=5000 V_{0}$ for (a) $8 \times 8$ linear elements, (b) $4 \times 4$ quadratic elements, and (c) $8 \times 8$ NURBS elements. The color shows the area stretch $J$ displayed as $\log _{10} J$.

augmented Lagrange multiplier method. In principle, any 3D contact algorithm can be applied straight forwardly to the proposed membrane formulation. Fig. 9 shows the deformation of balloon and cushion for various gravity levels. As shown, the deformation becomes very large, which makes the problem very challenging. The example is interesting as it involves large deformations, contact, pressure loading, hydrostatic loading and two volume constraints.

\subsection{Growth of a hemispherical water droplet}

As a validation of the formulation for liquid membranes, we consider the growth of a hemispherical droplet resting on a rigid substrate and maintaining a contact angle of $90^{\circ}$. The problem is similar to the balloon inflation example (Sec. 4.1), and the same FE meshes are used. For a liquid water membrane the membrane stress is given by Eq. (33), i.e. the stress is deformation independent. This implies that only the out-of-plane but not the in-plane forces provide stiffness and the formulation is unstable in itself. The formulation can be stabilized by adding deformation dependent in-plane forces through Eq. 65.1). These forces should not influence the out-of-plane behavior such that the original liquid membrane formulation remains 


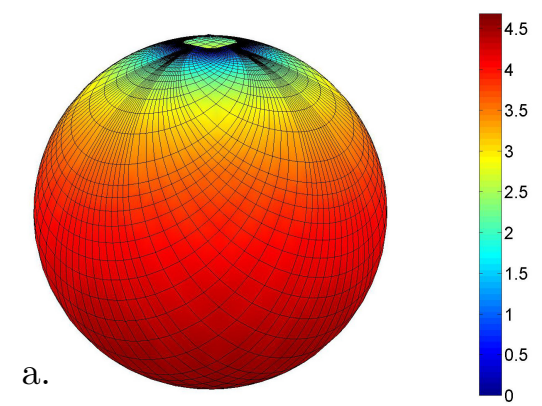

b.

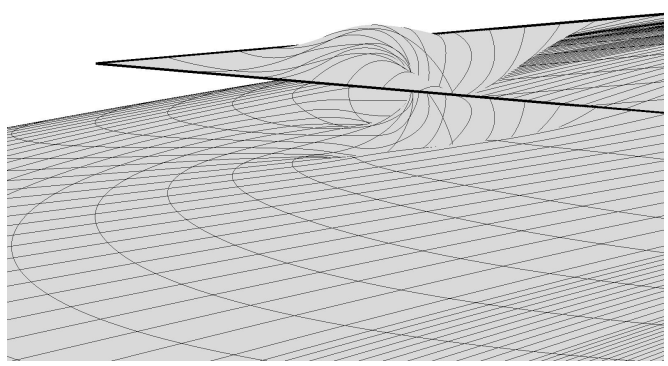

Figure 8: Inflated square sheet: deformation at $V=5000 V_{0}$ for $88 \times 88$ NURBS elements: (a) overall deformation, (b) deformation at the corner.

unaffected. We simply use the incompressible Neo-Hookean model to provide the additional in-plane stability. The Neo-Hookean material parameter $\mu$ then becomes a numerical stability parameter that should not affect the physical results. The internal forces acting on the finite element nodes are then simply given by

$$
\mathbf{f}_{\text {int }}^{e}=\mathbf{f}_{\text {int }}^{e}\left(\sigma_{\text {liquid }}^{\alpha \beta}\right)+\mathbf{f}_{\text {inti }}^{e}\left(\sigma_{\text {solid }}^{\alpha \beta}\right) .
$$

For this example, like in Sec. 4.1, the pressure-volume relation is also know analytically. Setting $\sigma=p r / 2=\gamma$, with $r=\lambda R$ and $V=\lambda^{3} V_{0}$, we find

$$
\frac{p R}{\gamma}=2\left(\frac{V_{0}}{V}\right)^{\frac{1}{3}} .
$$

Since the pressure remains positive (and is thus stabilizing the structure) the volume can also be decreased. The computed pressure and the convergence of the proposed finite element formulation to the analytical result are shown in Fig. 10. Several values for the numerical stability parameter $\mu$ are considered. They all converge to the desired analytical result. Quadratic Lagrange and NURBS elements are considered, and it is seen that the NURBS formulation converges much faster. This is attributed to the higher surface continuity that appears in $\mathbf{f}_{\text {inti }}^{e}$ according to Eq. 65.1). Decreasing $\mu$ improves the accuracy. A more detailed analysis of the model proposed in $\mathrm{Eq}(78)$ along with the effect of parameter $\mu$ is required and will be considered in the future.

\subsection{Liquid droplet on a rigid substrate}

The last example examines a static water droplet in contact with a rigid substrate. A distinct feature of liquid membranes is that they can form sharp contact angles at the contact boundary. In this case, the membrane surface forms a kink at the contact boundary. These surface discontinuities are associated with out-of-plane line forces. Such forces are not considered in the present framework. We thus consider a contact angle of $180^{\circ}$. Initially, prior to loading and contact, the droplet is spherical. We denote the initial radius $R$, and the initial volume $V_{0}:=4 \pi R^{3} / 3$. The water inside the droplet is considered incompressible such that the volume remains constant during deformation. The weight of the water causes hydrostatic pressure loading of the membrane leading to contact with the substrate. Fig. 11 shows the computed droplet deformation for various gravity values considering different FE formulations. As the 

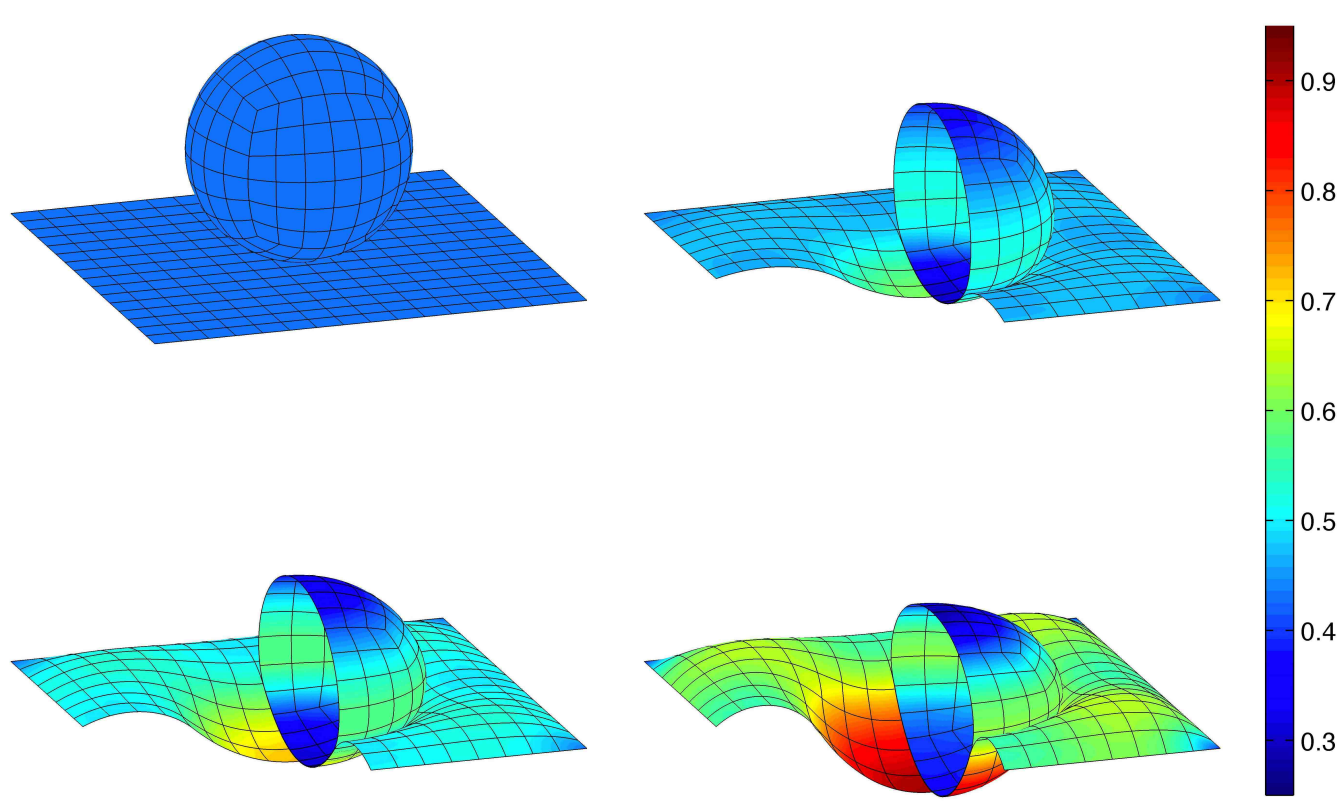

Figure 9: Cushion contact: configurations at $\rho g=\{0,0.2,0.4,0.8\} \mu / R$. The coloring shows the stress invariant $I_{1}=\operatorname{tr} \sigma=\sigma_{\alpha}^{\alpha}$.

gravity level increases the droplet spreads out on the substrate. Formulation (78) is used for the computations. Stability parameter $\mu$ is set to $0.005 \gamma$. For this $\mu$, the errors in the surface tension are less than $2.5 \%$ in the case of quadratic Lagrange elements (Fig 11, top.) and less than $1 \%$ in the case of cubic T-splines (Fig 11, bottom). In both cases the errors increase along with $\rho g$. In the case of Lagrange elements the largest errors are found at the element boundaries, where the formulation is only $C^{0}$-continous. For the T-spline case, which is $C^{2}$ continous over the entire surface (appart from two degenerate points at the top and bottom), the error is uniformly spread over the surface. It is remarked that the presented droplet model is much more general than the classical FE droplet formulation of Brown et al. (1980). This is discussed in detail in a forthcoming publication.

\section{Conclusion}

A novel computational formulation that is suitable for both solid and liquid, i.e. surface-tensiondriven, membranes is presented. The theory, outlined in Sec. 2, is based on the differential geometry of curved surfaces, allowing for a very general formulation that accounts for large deformations and general material laws. Curvilinear coordinates are used to formulate the surface geometry, kinematics, constitution, and balance laws. The governing strong and weak forms are split into the in-plane and out-of-plane parts, allowing the use of different approximation techniques for both parts and an elegant treatment of liquid membranes. Also, the consideration of deformation-dependent pressure loading comes naturally within the proposed formulation. Various constraints imposed upon the membranes can be handled by the theory, including volume, area, and contact constraints.

The membrane formulation is discretized using nonlinear finite elements. This results in a very efficient formulation that only uses three degrees of freedom per surface node and avoids the use of local cartesian coordinate systems and the transformation of derivatives. This is discussed in Sec. 3 . 

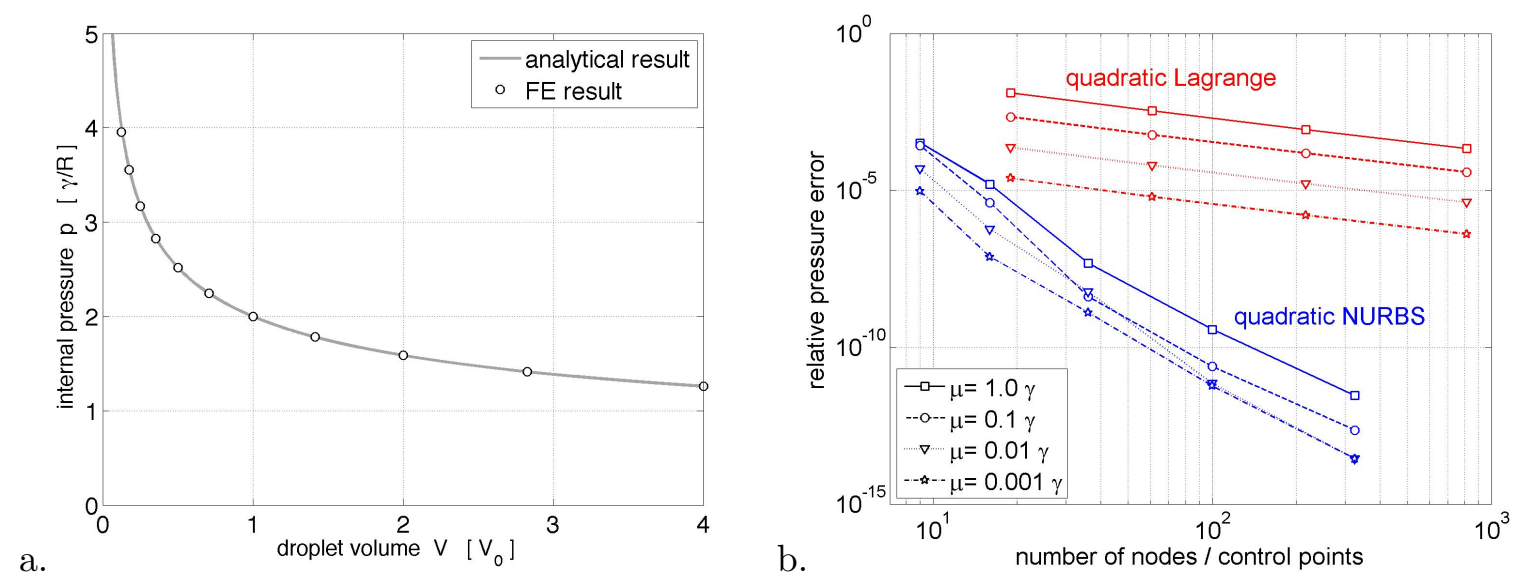

Figure 10: Growing liquid droplet: (a) pressure-volume relation for $V \in[1 / 84] V_{0}$ (FE result for 12 quadratic FE with $\mu=0.01 \gamma$ ); (b) convergence behavior for $V=4 V_{0}$ and various $\mu$, considering $3 \times 3$ Gaussian quadrature points.

The capabilities of the formulation are demonstrated by several challenging examples in Sec. 4 . Linear Lagrange, quadratic Lagrange, quadratic NURBS, and cubic T-spline finite elements are considered for the discretization. Constraints are imposed using the Lagrange multiplier method in the case of volume constraints and the penalty method for contact constraints. The inflation of a balloon and the growth of a droplet are used to validate the solid and liquid membrane formulations and they both yield excellent results. Comparing the different finite element types, the examples show that large accuracy gains lie between linear and quadratic Lagrange, and between quadratic Lagrange and isogeometric finite elements.

The presented membrane formulation has been successfully applied to liquid droplets in this paper, but a rigorous analysis is still needed to assess the approach proposed in Eq. (78). Another important extension to the present formulation is the inclusion of bending stiffness, which can be present in both fluid and solid films. In the latter case this should lead naturally to a rotation-free shell formulation, which can be suitably handled by isogeometric finite elements. A further interesting extension is the consideration and development of different membrane material laws. Such a development is especially important for the case of biological membranes, which are often characterized by complex material behavior.

\section{A Consistent linearization of various quantities}

For Newton's method we need to linearize the kinematical quantities of the discrete system at $\mathbf{x}$ in the direction $\Delta \mathbf{x}$. This is done at the FE level.

\section{A.1 Linearization of $a_{\alpha}$}

According to Eq. (55) we have

$$
\Delta \boldsymbol{a}_{\alpha}=\mathbf{N}_{, \alpha} \Delta \mathbf{x}_{e} .
$$


a.

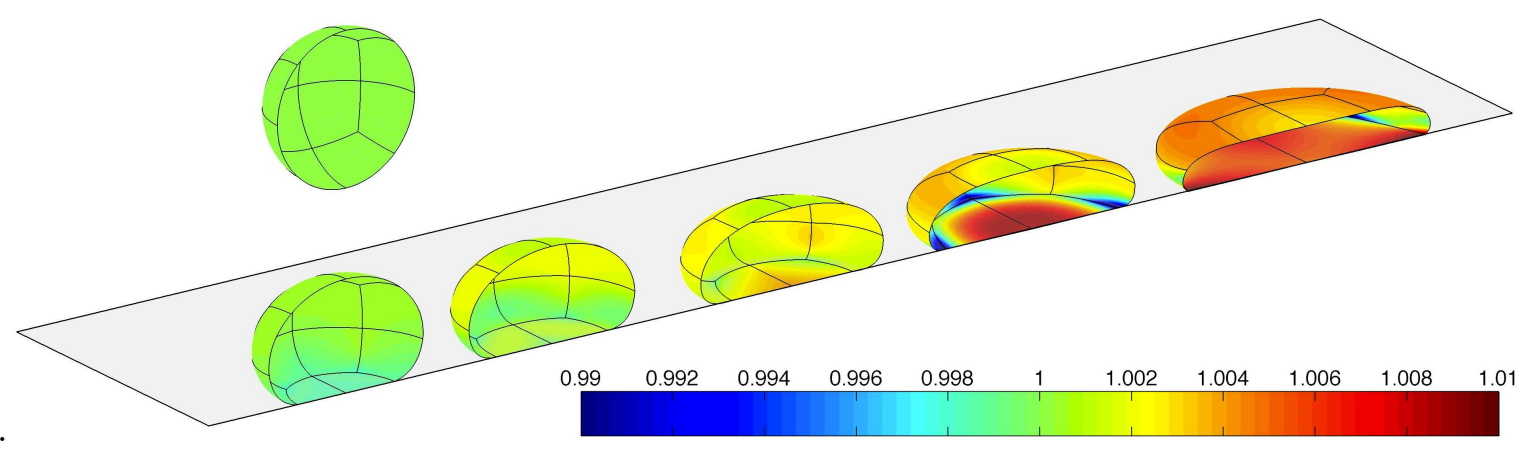

b.

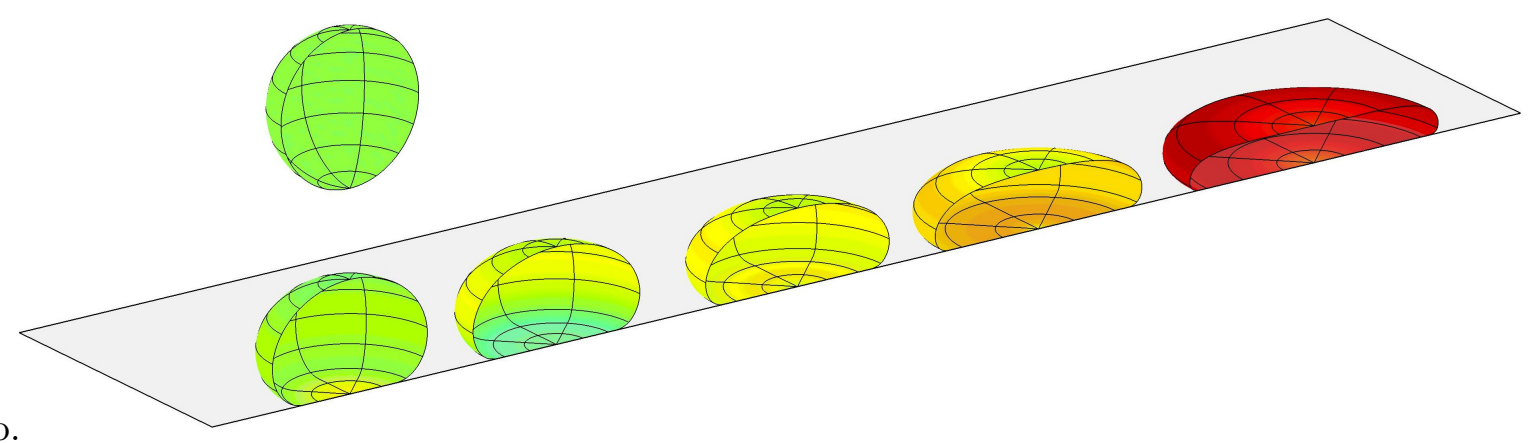

Figure 11: Liquid droplet contact: droplet deformation for $\rho g=\{1,2,4,8,20\} \gamma / R^{2}$, each with identical volume and $\mu=0.005 \gamma$ : (a) quadratic Lagrange elements, (b) cubic T-spline elements. The color shows the membrane stress $I_{1} / 2$ normalized by the surface tension $\gamma$. In theory $I_{1} /(2 \gamma)=1$.

\section{A.2 Linearization of $a_{\alpha \beta}$}

With definition (3) follows

$$
\Delta a_{\alpha \beta}=\left(\boldsymbol{a}_{\alpha} \cdot \mathbf{N}_{, \beta}+\boldsymbol{a}_{\beta} \cdot \mathbf{N}_{, \alpha}\right) \Delta \mathbf{x}_{e} .
$$

\section{A.3 Linearization of $J$}

The change $\Delta J$ can be written as

$$
\Delta J=\frac{\partial J}{\partial \boldsymbol{a}_{\alpha}} \cdot \Delta \boldsymbol{a}_{\alpha},
$$

where

$$
\frac{\partial J}{\partial \boldsymbol{a}_{\alpha}}=J \boldsymbol{a}^{\alpha}
$$

Thus

$$
\Delta J=J \boldsymbol{a}^{\alpha} \cdot \mathbf{N}_{, \alpha} \Delta \mathbf{x}_{e} .
$$

\section{A.4 Linearization of $a^{\alpha \beta}$}

From Eq. (5) and the formula

$$
a^{\alpha \beta}=\frac{1}{a} e^{\alpha \gamma} a_{\gamma \delta} e^{\delta \beta}, \quad a:=\operatorname{det} a_{\alpha \beta},
$$


where

$$
\left[e^{\alpha \beta}\right]=\left[\begin{array}{cc}
0 & 1 \\
-1 & 0
\end{array}\right]
$$

is the unit alternator, we find

$$
\Delta a^{\alpha \beta}=m^{\alpha \beta \gamma \delta} \boldsymbol{a}_{\gamma} \cdot \mathbf{N}_{, \delta} \Delta \mathbf{x}_{e}
$$

with

$$
m^{\alpha \beta \gamma \delta}=\frac{1}{a}\left(e^{\alpha \gamma} e^{\beta \delta}+e^{\alpha \delta} e^{\beta \gamma}\right)-2 a^{\alpha \beta} a^{\gamma \delta} .
$$

\section{A.5 Linearization of $n \mathrm{~d} a$}

The surface normal $\boldsymbol{n}$ appears together with the area element $\mathrm{d} a$ and it is convenient to linearize them together. According to Eqs. (6) and (20) we have

$$
\boldsymbol{n} \mathrm{d} a=\boldsymbol{a}_{1} \times \boldsymbol{a}_{2} \mathrm{~d} \square .
$$

Hence

$$
\Delta(\boldsymbol{n} \mathrm{d} a)=\sum_{I}\left(N_{, 1} \Delta \boldsymbol{x}_{I} \times \boldsymbol{a}_{2}+\boldsymbol{a}_{1} \times N_{, 2} \Delta \boldsymbol{x}_{I}\right) \mathrm{d} \square .
$$

Expanding $\Delta \boldsymbol{x}_{I}$ into $\Delta \boldsymbol{x}_{I}=\Delta x_{I}^{\alpha} \boldsymbol{a}_{\alpha}+\Delta x_{I}^{\mathrm{n}} \boldsymbol{n}$ we find

$$
\begin{aligned}
& \Delta \boldsymbol{x}_{I} \times \boldsymbol{a}_{2}=J_{a}\left(\boldsymbol{n} \Delta x_{I}^{1}-\boldsymbol{a}^{1} \Delta x_{I}^{\mathrm{n}}\right)=J_{a}\left(\boldsymbol{n} \otimes \boldsymbol{a}^{1}-\boldsymbol{a}^{1} \otimes \boldsymbol{n}\right) \Delta \boldsymbol{x}_{I}, \\
& \boldsymbol{a}_{1} \times \Delta \boldsymbol{x}_{I}=J_{a}\left(\boldsymbol{n} \Delta x_{I}^{2}-\boldsymbol{a}^{2} \Delta x_{I}^{\mathrm{n}}\right)=J_{a}\left(\boldsymbol{n} \otimes \boldsymbol{a}^{2}-\boldsymbol{a}^{2} \otimes \boldsymbol{n}\right) \Delta \boldsymbol{x}_{I},
\end{aligned}
$$

where $J_{a}=\sqrt{\operatorname{det} a_{\alpha \beta}}=\mathrm{d} a / \mathrm{d} \square$. Thus

$$
\Delta(\boldsymbol{n} \mathrm{d} a)=\left(\boldsymbol{n} \otimes \boldsymbol{a}^{\alpha}-\boldsymbol{a}^{\alpha} \otimes \boldsymbol{n}\right) \mathbf{N}_{, \alpha} \Delta \mathbf{x}_{e} \mathrm{~d} a .
$$

\section{A.6 Linearization of $\tau^{\alpha \beta}$}

For the solid model according to Eq. (32) we have

$$
\Delta \tau^{\alpha \beta}=\mu T\left(2 J^{-3} \Delta J a^{\alpha \beta}-J^{-2} \Delta a^{\alpha \beta}\right),
$$

which can be rewritten into

$$
\Delta \tau^{\alpha \beta}=c^{\alpha \beta \gamma \delta} \boldsymbol{a}_{\gamma} \cdot \mathbf{N}_{, \delta} \Delta \mathbf{x}_{e},
$$

with

$$
c^{\alpha \beta \gamma \delta}=\mu T J^{-2}\left(4 a^{\alpha \beta} a^{\gamma \delta}-\frac{1}{a}\left(e^{\alpha \gamma} e^{\beta \delta}+e^{\alpha \delta} e^{\beta \gamma}\right)\right) .
$$

Note that the tensor $\left[c^{\alpha \beta \gamma \beta}\right]$, like $\left[m^{\alpha \beta \gamma \beta}\right]$, posses both major and minor symmetries.

For the liquid model according to Eq. (33) we have

$$
\Delta \tau^{\alpha \beta}=\gamma\left(\Delta J a^{\alpha \beta}+J \Delta a^{\alpha \beta}\right),
$$

which can also be written in the form (94), where now

$$
c^{\alpha \beta \gamma \delta}=\gamma J\left(\frac{1}{a}\left(e^{\alpha \gamma} e^{\beta \delta}+e^{\alpha \delta} e^{\beta \gamma}\right)-a^{\alpha \beta} a^{\gamma \delta}\right) .
$$




\section{B Finite element tangent matrices}

\section{B.1 Tangent matrix associated with $G_{\text {int }}^{e}$}

The internal force vector $\mathbf{f}_{\text {int }}^{e}$, given in Eq. (63), yields

$$
\Delta \mathbf{f}_{\text {int }}^{e}=\int_{\Omega_{0}^{e}} \mathbf{N}_{, \alpha}^{T} \Delta \tau^{\alpha \beta} \mathbf{N}_{, \beta} \mathrm{d} A \mathbf{x}_{e}+\int_{\Omega_{0}^{e}} \mathbf{N}_{, \alpha}^{T} \tau^{\alpha \beta} \mathbf{N}_{, \beta} \mathrm{d} A \Delta \mathbf{x}_{e}
$$

In view of Eq. 94, we can write

$$
\Delta \mathbf{f}_{\mathrm{int}}^{e}=\left(\mathbf{k}_{\mathrm{mat}}^{e}+\mathbf{k}_{\mathrm{geo}}^{e}\right) \Delta \mathbf{x}_{e}
$$

where we have introduced the material stiffness matrix

$$
\mathbf{k}_{\mathrm{mat}}^{e}=\int_{\Omega_{0}^{e}} c^{\alpha \beta \gamma \delta} \mathbf{N}_{, \alpha}^{T}\left(\boldsymbol{a}_{\beta} \otimes \boldsymbol{a}_{\gamma}\right) \mathbf{N}_{, \delta} \mathrm{d} A
$$

and the geometric stiffness matrix

$$
\mathbf{k}_{\text {geo }}^{e}=\int_{\Omega_{0}^{e}} \mathbf{N}_{, \alpha}^{T} \tau^{\alpha \beta} \mathbf{N}_{, \beta} \mathrm{d} A
$$

Both these matrices are symmetric for the two constitutive models considered here. For those models, the terms in $\mathbf{k}_{\text {mat }}^{e}$ should be multiplied-out a-priory to obtain an efficient implementation. If we consider splitting $\mathbf{f}_{\text {int }}^{e}$ into $\mathbf{f}_{\text {inti }}^{e}$ and $\mathbf{f}_{\text {into }}^{e}$ additional stiffness terms are picked up. These are reported in a forthcoming publication.

\section{B.2 Tangent matrix associated with $G_{\mathrm{ext}}^{e}$}

From Eq. 67), for dead $\boldsymbol{f}_{0}$ and $\overline{\boldsymbol{t}}$, we have

$$
\Delta \mathbf{f}_{\mathrm{ext}}^{e}=\int_{\Omega^{e}} \mathbf{N}^{T} \boldsymbol{n} \Delta p \mathrm{~d} a+\int_{\Omega^{e}} \mathbf{N}^{T} p \Delta(\boldsymbol{n} \mathrm{d} a) .
$$

The first term is only required for hydrostatic loading according to Eq. (74). Here we find

$$
\Delta p=-\rho \boldsymbol{g} \mathbf{N} \Delta \mathbf{x}_{e} .
$$

Contribution $\Delta(\boldsymbol{n} \mathrm{d} a)$ is given by Eq. (92). As a result,

$$
\mathbf{k}_{\text {ext }}^{e}=-\int_{\Omega^{e}} \rho \mathbf{N}^{T} \boldsymbol{n} \otimes \boldsymbol{g} \mathbf{N} \mathrm{d} a+\int_{\Omega^{e}} p \mathbf{N}^{T}\left(\boldsymbol{n} \otimes \boldsymbol{a}^{\alpha}-\boldsymbol{a}^{\alpha} \otimes \boldsymbol{n}\right) \mathbf{N}_{, \alpha} \mathrm{d} a .
$$

\section{B.3 Tangent contributions associated with the volume constraint}

If the volume constraint $g_{\mathrm{v}}=0$ is active, we need to account for the unknown Lagrange multiplier $p_{\mathrm{v}}$ in the linearization. For the external forces we now have

$$
\Delta \mathbf{f}_{\mathrm{ext}}^{e}=\mathbf{k}_{\mathrm{ext}}^{e} \Delta \mathbf{x}_{e}+\mathbf{l}_{\mathrm{ext}}^{e} \Delta p_{\mathrm{v}}
$$

with

$$
\mathbf{l}_{\mathrm{ext}}^{e}=\frac{\partial \mathbf{f}_{\mathrm{ext}}^{e}}{\partial p_{\mathrm{v}}}=\int_{\Omega^{e}} \mathbf{N}^{T} \boldsymbol{n} \mathrm{d} a
$$


Further, at the element level,

$$
\Delta g_{\mathrm{v}}^{e}=\mathbf{h}_{\mathrm{v}}^{e} \Delta \mathbf{x}_{e}
$$

with

$$
\mathbf{h}_{\mathrm{v}}^{e}=\frac{\partial g_{\mathrm{v}}^{e}}{\partial \mathbf{x}_{e}}=\frac{1}{3} \int_{\Omega^{e}} \boldsymbol{n} \cdot \mathbf{N} \mathrm{d} a+\frac{1}{3} \int_{\Omega^{e}} \boldsymbol{x} \cdot\left(\boldsymbol{n} \otimes \boldsymbol{a}^{\alpha}-\boldsymbol{a}^{\alpha} \otimes \boldsymbol{n}\right) \mathbf{N}_{, \alpha} \mathrm{d} a .
$$

The preceding contributions can be arranged into the elemental tangent matrix

$$
\mathbf{k}^{e}:=\left[\begin{array}{cc}
\mathbf{k}_{\mathrm{int}}^{e}-\mathbf{k}_{\mathrm{ext}}^{e} & -\mathbf{l}_{\mathrm{ext}}^{e} \\
\mathbf{h}_{\mathrm{v}}^{e} & 0
\end{array}\right],
$$

which describes the change in $\mathbf{f}_{\mathrm{int}}-\mathbf{f}_{\mathrm{ext}}$ and $g_{\mathrm{v}}$ due to changes in position $\mathbf{x}_{e}$ and pressure $p_{\mathrm{v}}$.

\section{Acknowledgements}

The authors are grateful to the German Research Foundation (DFG) for supporting this research under projects SA1822/3-2, SA1822/5-1 and GSC 111.

\section{References}

Ambroziak, A. and Klosowski, P. (2006). A four-node 3D isoparametric membrane element. Task Quarterly, 10(1):35-47.

Arciniega, R. A. and Reddy, J. N. (2007). Tensor-based finite element formulation for geometrically nonlinear analysis of shell structures. Comput. Methods Appl. Mech. Engrg., 196(4-6):1048-1073.

Benson, D. J., Bazilevs, Y., Hsu, M.-C., and Hughes, T. J. R. (2011). A large deformation, rotation-free, isogeometric shell. Comp. Methods Appl. Mech. Engrg., 200(13-16):1367-1378.

Bletzinger, K.-U., Wüchner, R., Daoud, F., and Camprubi, N. (2005). Computational methods for form finding and optimization of shells and membranes. Comput. Meth. Appl. Mech. Engrg., 194(30-33):3438-3452.

Bonet, J., Wood, R. D., and Mahaney, J. (2000). Aspects of the analysis of membrane structures. In Computational civil and structural engineering, pages 105-113. Civil-Comp. Press.

Borden, M. J., Scott, M. A., Evans, J. A., and Hughes, T. J. R. (2011). Isogeometric finite element data structures based on bezier extraction of NURBS. Int. J. Numer. Meth. Engng., $87: 15-47$.

Brown, R. A., Orr, F. M., and Scriven, L. E. (1980). Static drop on an inclined plate: Analysis by the finite element method. J. Colloid Interface Sci., 73(1):76-87.

Bufler, H. (1984). Pressure loaded structures under large deformations. J. Appl. Math. Mech., 64(7):287-295.

Contri, P. and Schrefler, B. (1988). A geometrically nonlinear finite element analysis of wrinkled membrane surfaces by a no-compression material model. Commun. Appl. Numer. M., 1(4):515 . 
De Lorenzis, L., Temizer, I., Wriggers, P., and Zavarise, G. (2011). A large deformation frictional contact formulation using NURBS-based isogeometric analysis. Int. J. Numer. Meth. Engng., 87:1278-1300.

Dung, N. T. and Wells, G. N. (2008). Geometrically nonlinear formulation for thin shells without rotation degrees of freedom. Comput. Methods Appl. Mech. Engrg., 197:2778-2788.

Flores, F. G. and Estrada, C. F. (2007). A rotation-free thin shell quadrilateral. Comput. Methods Appl. Mech. Engrg., 196(25-28):2631-2646.

Fried, I. (1982). Finite element computation of large rubber membrane deformations. Int. J. Numer. Mech. Engng., 18:653-660.

Gosling, P. and Lewis, W. (1996). Optimal structural membranesi. formulation of a curved quadrilateral element for surface definition. Comput. Struct., 61(5):885-895.

Gurtin, M. E. and Murdoch, I. (1975). A continuum theory of elastic material surfaces. Arch. Rat. Mech. Anal., 57(4):291-323.

Haseganu, E. M. and Steigmann, D. J. (1994). Analysis of partly wrinkled membranes by the method of dynamic relaxation. Comput. Mech., 14(6):596-614.

Ibrahimbegovic, A. and Gruttmann, F. (1993). A consistent finite element formulation of nonlinear membrane shell theory with particular reference to elastic rubberlike material. Finite Elem. Anal. Des., 13(1):75-86.

Kiendl, J., Bazilevs, Y., Hsu, M.-C., Wüchner, R., and Bletzinger, K.-U. (2010). The bending strip method for isogeometric analysis of kirchhoff-love shell structures comprised of multiple patches. Comput. Methods Appl. Mech. Engrg., 199(37-40):2403-2416.

Kloeppel, T. and Wall, W. A. (2011). A novel two-layer, coupled finite element approach for modeling the nonlinear elastic and viscoelastic behavior of human erythrocytes. Biomech. Model. Mechanobiol., 10(4):445-459.

Linhard, J., Wüchner, R., and Bletzinger, K.-U. (2007). "Upgrading" membranes to shells The CEG rotation free element and its application in structural anaylsis. Finite Elem. Anal. Des., 44(1-2):63-74.

Lu, K., Accorsi, M., and Leonard, J. (2001). Finite element analysis of membrane wrinkling. Int. J. Numer. Meth. Engng., 50(5):1017-1038.

Manh, N. D., Evgrafov, A., Gersborg, A. R., and Gravesen, J. (2011). Isogeometric shape optimization of vibrating membranes. Comp. Meth. Appl. Mech. Engrg., 200(13-16):13431353.

Muttin, F. (1996). A finite element for wrinkled curved elastic membranes, and its application to sails. Comm. Numer. Meth. Engng., 12:775-785.

Nguyen-Thanh, N., Kiendl, J., Nguyen-Xuan, H., Wüchner, R., Bletzinger, K.-U., Bazilevs, Y., and Rabczuk, T. (2011). Rotation free isogeometric thin shell analysis using pht-splines. Comput. Methods Appl. Mech. Engrg., 200(47-48):3410-3424.

Oden, J. T. (2006). Finite Elements of Nonlinear Continua. Dover Edition.

Oden, J. T. and Sato, T. (1967). Finite strains and displacements of elastic membranes by the finite element method. Int. J. Solids Struct., 3(4):471-488. 
Roddeman, D., Drukker, J., Oomens, C., and Janssen, J. (1987). The wrinkling of thin membranes: Part II. Nnumerical analysis. J. Appl. Mech., 54(4):888.

Rumpel, T. and Schweizerhof, K. (2003). Volume dependent pressure loading and its influence on the stability of structures. Int. J. Numer. Meth. Engng., 56:211-238.

Sauer, R. A. (2011). Enriched contact finite elements for stable peeling computations. Int. J. Numer. Meth. Engrg., 87:593-616.

Sauer, R. A. (2012). Local finite element enrichment strategies for 2D contact computations and a corresponding postprocessing scheme. submitted to Comput. Mech.

Sauer, R. A. and De Lorenzis, L. (2012). A computational contact formulation based on surface potentials. Comput. Methods Appl. Mech. Engrg., in press.

Sauer, R. A. and Li, S. (2007). An atomic interaction-based continuum model for adhesive contact mechanics. Finite Elem. Anal. Des., 43(5):384-396.

Schweizerhof, K. and Ramm, E. (1984). Displacement dependent pressure loads in nonlinear finite element analyses. Comput. Struct., 18(6):1099-1114.

Scott, M. A., Borden, M. J., Verhoosel, C. V., Sederberg, T. W., and Hughes, T. J. R. (2011). Isogeometric finite element data structures based on Bézier extraction of T-splines. Int. J. Numer. Meth. Engng., 88(2):126-156.

Stanuszek, M. (2003). FE analysis of large deformations of membranes with wrinkling. Finite. Elem. Anal. Des., 39(7):599-618.

Steigmann, D. J. (1999). On the relationship between the Cosserat and Kirchhoff-Love theories of elastic shells. Math. Mech. Solids, 4:275-288.

Tang, S. C. (1982). Large strain analysis of an inflating membrane. Comput. Struct., 15(1):7178.

Temizer, I., Wriggers, P., and Hughes, T. J. R. (2012). Three-dimensional mortar-based frictional contact treatment in isogeometric analysis with NURBS. Comput. Methods Appl. Mech. Engrg., 209-212:115-128.

Weinberg, K. and Neff, P. (2008). A geometrically exact thin membrane model - investigation of large deformation and wrinkling. Int. J. Numer. Meth. Engng., 74:871-893.

Wriggers, P. (2006). Computational Contact Mechanics. Springer, $2^{\text {nd }}$ edition.

Wriggers, P. (2008). Nonlinear Finite Element Methods. Springer.

Wriggers, P. and Taylor, R. L. (1990). A fully non-linear axisymmetrical membrane element for rubber-like materials. Engrg. Comput., 7(1):303-310.

Wu, B., Du, X., and Tan, H. (1996). A three-dimensional FE nonlinear analysis of membranes. Comput Struct., 59(4):1-5.

Youn, S.-K. and Lee, E.-S. (2006). Finite element analysis of wrinkling membrane structures with large deformations. Finite Elem. Anal. Des., 42(8-9):780-791. 